DFTT 3/2007

QMUL-PH-06-13

\title{
The twisted open string partition function and Yukawa couplings
}

\author{
Rodolfo Russo \\ Centre for Research in String Theory \\ Department of Physics \\ Queen Mary, University of London \\ Mile End Road, London, E1 4NS, United Kingdom \\ Stefano Sciuto \\ Dipartimento di Fisica Teorica, Università di Torino \\ and INFN, Sezione di Torino \\ Via P. Giuria 1, I-10125 Torino, Italy
}

\begin{abstract}
We use the operator formalism to derive the bosonic contribution to the twisted open string partition function in toroidal compactifications. This amplitude describes, for instance, the planar interaction between $g+1$ magnetized or intersecting D-branes. We write the result both in the closed and in the open string channel in terms of Prym differentials on the appropriate Riemann surface. Then we focus on the $g=2$ case for a 2-torus. By factorizing the twisted partition function in the open string channel we obtain an explicit expression for the 3-twist field correlator, which is the main ingredient in the computation of Yukawa couplings in D-brane phenomenological models. This provides an alternative method for computing these couplings that does not rely on the stress-energy tensor technique.
\end{abstract}




\section{Introduction}

It is well known that various interesting string vacua are described by free Conformal Field Theories (CFT) with twisted (i.e. periodic up to a phase) boundary conditions on the world-sheet fields. This happens in the case of orbifolds for closed strings [1], and, with the doubling trick, also for the open strings stretched between intersecting [2] or magnetized D-branes [3]. In these cases the 2-dimensional equations of motions take the usual form of the free wave equation, but the boundary conditions on the world-sheet fields can change; in particular this happens in the interactions between closed strings belonging to different twisted sectors or open strings stretched between different D-branes. The CFT operators implementing a change in the boundary conditions for the bosonic coordinates are called twist fields and are rather complicated objects from the world-sheet point of view. In the following we will use $\sigma_{\epsilon}$ to indicate a twists field changing the boundary conditions of a complexified bosonic coordinate by a phase $\mathrm{e}^{2 \pi i \epsilon}$. In superstring theories, a similar pattern is present also in the fermionic sector; however in this case the operators changing the boundary conditions (called spin fields) can be described in terms of simple free fields thanks to the bosonization equivalence. In this paper we will exclusively focus on the bosonic twist fields for which no simple free theory description is known.

The couplings among 3-twist fields are important in various context, in particular in string compactifications that display realistic phenomenological features, where they are directly related to the Yukawa couplings of the low energy effective action. The twist fields correlators are usually computed by using the techniques introduced in [4]. In the context of closed string theory on orbifolds these techniques were applied to the computation of Yukawa couplings in [5]. More recently other semi-realistic string vacua have been under intense study: in this case the standard model fields are realized by means of open strings stretched between intersecting or magnetized D-branes. From a CFT point of view, the main ingredient in the determination of the Yukawa couplings in these models is the evaluation of a 3-twist coupling. By following the prescription of [4], the twist-fields correlators in open string setups have been studied in detail 1 by [7, 8].

Another very important quantity that can be computed in string configurations with twisted boundary conditions is the partition function. This can be seen as a contribution to the cosmological constant and thus serves, in orbifold compactifications, as a check of whether supersymmetry is broken or not in the twisted sectors [9]. In open string configurations the partition function captures the D-brane dynamics and string coordinates with non-trivial monodromies appear when the D-branes support constant electro-magnetic fields [10] or have constant velocity [11. Twisted partition functions are of interest also in topological string theory and Heterotic strings, see for example [12, 13] for recent

\footnotetext{
${ }^{1}$ The first analysis of the twist fields correlators in the open string case was given in [6]
} 
applications.

In this paper we use the operator formalism [14, 15] to compute the twisted partition function for open bosonic strings in a generic toroidal compactification. For concreteness, we work in the setup of open strings stretched between magnetized D-branes, where the parameters $\epsilon_{i}$ depend on the magnetic fields on the D-branes. This setup is more general than the one usually encountered in orbifold compactifications, because the twists $\epsilon_{i}$ are generically non-rational numbers. In this paper we make two simplifying assumptions: we require that the D-branes wrap only once the geometrical torus and impose that the world-volume magnetic fluxes commute, see (3.1). While the first assumption provides just a technical simplification, the presence of non-abelian backgrounds would make the problem rather more difficult. We hope to relax these hypothesis in a future work.

We will start our computation in the closed string channel, where the main ingredient is the boundary state describing a magnetized D-brane (see [16] and Refs. therein). We follow the approach of [17] and build a surface with $g+1$ borders starting from a vertex describing the interaction among $g+1$ closed strings. The two main novelties in the present computation are the use of magnetized boundary states and the analysis of the contribution of winding or Kaluza-Klein modes present in the compact case. Then by using the results of [18, 19] we exploit the modular properties of string amplitudes to rewrite the same diagram with magnetized D-branes in the open string channel. All our expressions are written in terms of geometrical quantities that commonly appear in the study of Riemann surfaces, such as the period matrix or the Riemann class. In particular, in our case the Prym differentials, which are 1-forms with non-trivial monodromies, play a crucial role. The operator formalism naturally gives for all these quantities, including the Prym differentials [18], an explicit expression in terms of sums or products over the Schottky group. Even if we use as starting point a setup with magnetized D-branes, we can easily perform a T-duality at any step of the computation. So, in our approach, the computations in the intersecting and in the magnetized descriptions are essentially on the same footing. In principle, for rational twists $\epsilon_{i}$, an expression for the twisted partition function could be derived from the higher genus correlation functions studied in [20], but, to the best of our knowledge, this has not been done explicitly.

In a second part of the paper we specialize to the $T^{2}$ case for $g=2$ and show explicitly that the twisted partition function can be used also to derive the correlators among three twist fields and thus the Yukawa couplings in models with magnetized D-branes. The idea is simple: instead of following [4] and factorizing the 4-point tree-level amplitude, we derive the couplings among twist fields by factorizing the planar 2-loop vacuum diagram in the open string channel as depicted in Fig. 1. This alternative option was already proposed in [21], where the formal properties of higher loop amplitudes were studied. However this program has not been carried out explicitly in any concrete case and probably the main 


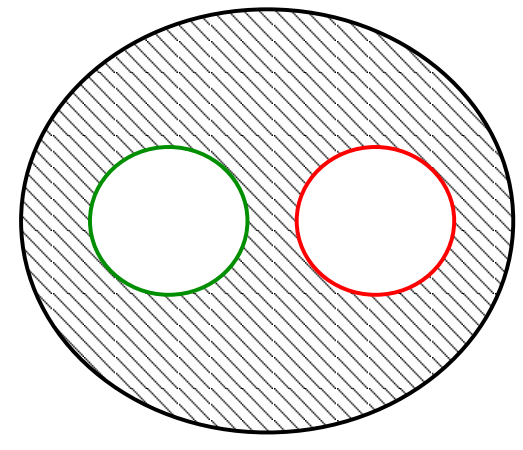

17.

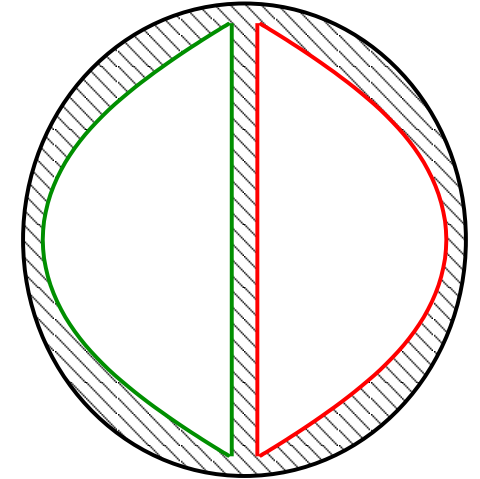

10

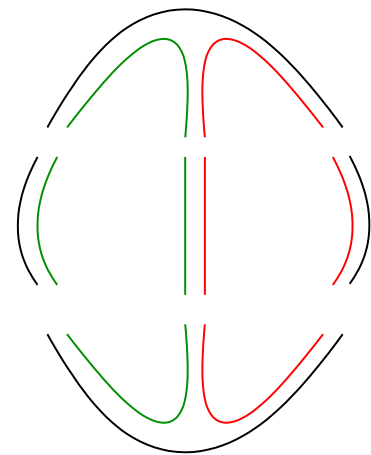

15

Figure 1: 19 represents the twisted 2-loop partition function in the open string channel in a generic point of the world-sheet moduli space: on the three borders there are different magnetic fields $F_{i}$; $1 \mathrm{~b}$ is the degeneration limit we are interested in, which is obtained by focusing on the corner of the world-sheet moduli space defined in (4.1); 1 ; is the factorization of $1 \mathrm{~b}$ into two twisted 3-string vertices and three propagators: this is obtained by focusing only on the leading term in the expansion of the previous point .

stumbling point has been so far the lack of explicit enough formulae for the multiloop twisted partition function itself. At first sight, the idea of factorizing loop instead of tree amplitudes may seem an unnecessary complication. Actually it turns out that for certain purposes it is technically easier to follow this path. For instance, in the case of the multiloop partition function, it is easier to control the overall $\epsilon_{i}$-dependent normalization, since it is directly related to the tension of the D-branes. Moreover the computation of the partition function in our formalism is completely algorithmic and does not require any space-time intuition. In fact we will derive the Yukawa couplings directly in a type IIB setup, thus providing, without the need of any T-duality, a string counterpart of the field theory computation of Ref. [22]. However, as we already said, T-dualities can be easily performed in our approach. So one can see that, in a type IIA setup, the zeromode contribution to the partition function depends on the Kähler moduli and, after factorization, reproduces exactly the world-sheet instanton contribution to the Yukawa couplings for intersecting brane configurations.

The structure of the paper is the following. In Section 2 we summarize the main properties of open and closed bosonic strings in toroidal compactification and fix our conventions, see [23, 24] for more details. In Section 3 we generalize the approach of [19, 25] and compute the twisted partition function on a $T^{2 d}$ for a planar orientable Riemann surface with $g+1$ borders and no handles. We first work in the closed string channel where the amplitudes is obtained by sewing together $g+1$ boundary states on a sphere. 
Then we perform a modular transformation and translate the result in the open string channel. This is done by using the generalization of the product formulae for the Theta functions derived in [18. In Section 4 we focus on the $g=2$ partition function for a 2 -torus and study the limit where the surface degenerates in two disks connected by three open string propagators. As suggested by Fig. 1, this allows us to derive the 3-string coupling among twisted states. We first consider the non-zero mode contribution which is the only one present in the uncompact space. The contribution to the 3 -twist couplings derived in this way is usually indicated as the quantum (from the world-sheet point of view) part. Then we study the factorization of the full twisted partition function on $T^{2}$. In this way we obtain the full 3-twist couplings, including what is usually called the classical contribution. Our results for these couplings are in agreement with previous works [5, 7, 8, 22], thus providing a very non-trivial test for the expression of the twisted partition function we obtained.

\section{Open and closed strings on magnetized tori}

The setup we consider is that of a toroidal compactification $T^{2 d}$ of bosonic string theory with $g+1$ space filling D-branes labeled by the index $i=0, \ldots, g$. The closed strings feel the standard geometrical metric $G_{M N}$ and the NS-NS $B_{M N}$ field that we will take to be constant. Our coordinates indicated with the indices $M, N, \ldots$ are parallel to the lattice defining the torus and have period $2 \pi \sqrt{\alpha^{\prime}}$ (this is the so-called integral basis). The end-points of the open strings are charged under the gauge invariant combination $\mathcal{F}_{i}=B+2 \pi \alpha^{\prime} F_{i}$ between the $B$ field and the $U(1)$ magnetic field $F_{i}$ living on the D-brane considered. We restricts ourselves to the case where also the magnetic fields are constant so that the string world-sheet action is quadratic. Notice that, in the integral basis, the elements of $F_{i}$ are quantized

$$
F_{i M N}=\frac{1}{2 \pi \alpha^{\prime}} \frac{p_{i M N}}{l_{i M} l_{i N}}, \quad i=0,1, . ., g,
$$

where $l_{i M}$ is the winding number of the D-brane along the direction $M$ and $p_{i M N}$ is the magnetic flux in the plane $M N$. If an open string is stretched between the D-branes $k$ and $l$, with gauge fields $F_{k}$ and $F_{l}$, then the boundary conditions on the open string coordinates $x^{M}(z, \bar{z})$ are specified by the reflection matrix $2 R_{i}$ :

$$
\begin{gathered}
\left.\bar{\partial} x^{M}(z, \bar{z})\right|_{\sigma=0}=\left.\left(R_{k}\right)_{N}^{M} \partial x^{N}(z, \bar{z})\right|_{\sigma=0},\left.\quad \bar{\partial} x^{M}(z, \bar{z})\right|_{\sigma=\pi}=\left.\left(R_{l}\right)_{N}^{M} \partial x^{N}(z, \bar{z})\right|_{\sigma=\pi} \\
\text { with } \quad R_{i}=\left(G-\mathcal{F}_{i}\right)^{-1}\left(G+\mathcal{F}_{i}\right)
\end{gathered}
$$

\footnotetext{
${ }^{2}$ We follow the conventions of 26
} 
where, as usual, $z=\mathrm{e}^{\tau+\mathrm{i} \sigma}$. Then the mode expansion of $x^{M}(z, \bar{z})$ can be written in terms of a single meromorphic function $X^{M}(z)$ whose monodromy properties are encoded by the matrix $R_{l k}$ :

$$
\begin{gathered}
x^{M}(z, \bar{z})=q^{M}+\frac{1}{2}\left[X^{M}(z)+\left(R_{k}\right)_{N}^{M} X^{N}(\bar{z})\right] \\
X^{M}\left(\mathrm{e}^{2 \pi \mathrm{i}} z\right)=\left(R_{l k}\right)_{N}^{M} X^{N}(z), \quad \text { with } \quad R_{l k} \equiv R_{l}^{-1} R_{k} .
\end{gathered}
$$

When the gauge fields $F_{k}$ and $F_{l}$ are non-zero but equal, then we see that $X^{M}(z)$ is periodic and we are in the so-called non-commutative setup. In this case the zero-modes $q^{M}$ develop non-trivial commutation relations [27] proportional to $\left(R_{l}^{M N}-R_{l}^{N M}\right) / 4$ and in general all open strings modes are contracted with the open string metric: $G_{\text {open }}^{M N} \equiv$ $G^{M N} / 2+\left(R_{l}^{M N}+R_{l}^{N M}\right) / 4$. The multiloop partition function and amplitudes for bosonic strings in this background were computed in [28].

When $F_{k} \neq F_{l}$, the meromorphic function $X^{M}(z)$ has non-trivial monodromy properties encoded by $R_{l k}$. Since all $R_{i}$ 's are $2 d \times 2 d$ dimensional real matrices satisfying

$$
{ }^{\mathrm{t}} R_{i} G R_{i}=G, \quad i=0,1, . ., g,
$$

the eigenvalues of the monodromy matrix $R_{l k}$ are just pairs of complex conjugate numbers of norm 1. Thus these eigenvalues can be represented by phases and we can extract unambiguously $d$ independent parameters that will be indicated 3 by $\mathrm{e}^{2 \pi \mathrm{i} \epsilon_{l k}^{a}}, \quad a=1,2, \ldots, d$. The mode expansion of $X(z)$ is more easily written in the (complex) basis where the monodromy matrix is diagonal; we indicate the meromorphic fields in this basis by $\mathcal{Z}^{a}(z)$. The periodicity properties imply that for the string stretched between the branes $k$ and $l$ the modes are shifted by $\pm \epsilon_{l k}^{a}$ :

$$
\partial \mathcal{Z}^{a}(z)=-\mathrm{i} \sqrt{2 \alpha^{\prime}}\left(\sum_{n=1}^{\infty} \bar{\alpha}_{n-\epsilon_{l k}^{a}}^{a} z^{-n+\epsilon_{l k}^{a}-1}+\sum_{n=0}^{\infty} \alpha_{n+\epsilon_{l k}^{\dagger}}^{\dagger a} z^{n+\epsilon_{l k}^{a}-1}\right), \quad a=1,2, \ldots, d,
$$

with commutation relations

$$
\left[\bar{\alpha}_{n-\epsilon_{l k}^{a}}^{a}, \bar{\alpha}_{m-\epsilon_{l k}^{b}}^{\dagger b}\right]=\left(n-\epsilon_{l k}^{a}\right) \delta^{a b} \delta_{n, m}, \quad\left[\alpha_{n+\epsilon_{l k}^{a}}^{a}, \alpha_{m+\epsilon_{l k}^{b}}^{\dagger b}\right]=\left(n+\epsilon_{l k}^{a}\right) \delta^{a b} \delta_{n, m} .
$$

The vertex operators describing the physical states of these twisted open strings contain a product of twist fields $\prod_{a=1}^{d} \sigma_{\epsilon_{l k}^{a}}$, each one twisting the string coordinate defined by the corresponding $R_{l k}$ eigenvector. This implies that the open string spectrum depends on $G, B$ and the $F_{i}$ 's: for instance the first state (which is the usual open string tachyon in absence of fluxes) has a mass square given by

$$
\alpha^{\prime} M_{l k}^{2}=-1+\sum_{a=1}^{d} \epsilon_{l k}^{a}\left(1-\epsilon_{l k}^{a}\right) / 2
$$

\footnotetext{
${ }^{3}$ The sign ambiguity in the exponent can be fixed by requiring for instance $\epsilon_{01}^{a} \geq 0$.
} 
The $\epsilon$-dependent shift with the respect to the usual value is expected, since it is nothing else than the contribution to $L_{0}$ coming from the conformal weight of each twist field $\sigma_{\epsilon_{l k}^{a}}$. The zero-mode structure in this sector is somewhat subtle: the commutation relations are $\left[q^{M}, q^{N}\right]=\left(F^{-1}\right)^{M N}$ and this yields, in toroidal compactification, a finite dimensional degeneracy for each string state [3]. In fact the zero-mode part of the string vertex operators should encode the same information of the field theoretic wave-functions describing a charged particle on a magnetized torus, and in field theory one finds, for a fixed energy, finitely many inequivalent wave-functions [22]. In our approach we will bypass these subtleties and will not need to deal with the zero-mode structure of the open string vertices.

Going to the closed string sector, we will use the following conventions:

$x_{\mathrm{cl}}^{M}(z, \bar{z})=\frac{X_{\mathrm{cl}}^{M}(z)+\tilde{X}_{\mathrm{cl}}^{M}(\bar{z})}{2}$, where $X_{\mathrm{cl}}^{M}(z)=\mathrm{x}^{M}-\mathrm{i} \sqrt{2 \alpha^{\prime}} \alpha_{0}^{M} \ln z+\mathrm{i} \sqrt{2 \alpha^{\prime}} \sum_{m \neq 0} \frac{\alpha_{m}^{M} z^{-m}}{m}$,

with the commutation relations

$$
\left[\alpha_{m}^{M}, \alpha_{-n}^{N}\right]=n \delta_{n, m} G^{M N}, \quad \text { and } \quad\left[\mathrm{x}^{M}, \alpha_{0}^{N}\right]=\mathrm{i} \sqrt{2 \alpha^{\prime}} G^{M N}
$$

and similarly in the right-moving sector. When convenient, we will also use the normalized oscillators $a_{n}$ or $\tilde{a}_{n}$, with $\alpha_{n}=\sqrt{n} a_{n}, \alpha_{-n}=\sqrt{n} a_{n}^{\dagger}$ for $n>0$ and $\alpha_{0}=a_{0}$.

From the closed string point of view, the D-branes are described by boundary states $\left|B_{i}\right\rangle$, that enforce an identification between the left and the right moving modes (2.2). In order to write this identification in the closed string channel, it is convenient to map the upper-half complex plane $z$ into the circle of unit radius $w=-(z-\mathrm{i}) /(z+\mathrm{i})$. Thus we get

$$
\text { Eq. (2.2) }\left.\Rightarrow \bar{\partial} x^{M}(w, 1 / \bar{w})\right|_{\tau=0}=-\left.\left(R_{i}\right)^{M}{ }_{N} \partial x^{N}(w, 1 / \bar{w})\right|_{\tau=0} .
$$

At the level of closed string modes this condition reads

$$
\left[\left(G+\mathcal{F}_{i}\right)_{M N} \alpha_{n}^{N}+\left(G-\mathcal{F}_{i}\right)_{M N} \tilde{\alpha}_{-n}^{N}\right]\left|B_{i}\right\rangle=0, \quad \forall n \in Z
$$

Notice that the closed strings modes are integer as usual, and the magnetic fields $F_{i}$ enter only in the gluing conditions. Thus, contrary to what happens for the twisted open strings, the closed string spectrum is unaffected by the magnetic fields. In particular, the allowed winding and Kaluza-Klein modes are encoded in the Narain lattice which depends only on $G$ and $B$. In our conventions the eigenvalues of the operators $\alpha_{0}$ and $\tilde{\alpha}_{0}$ are

$$
\left(\alpha_{0}\right)^{M}=\frac{G^{M N}}{\sqrt{2}}\left[n_{N}+\left(G_{N N^{\prime}}-B_{N N^{\prime}}\right) m^{N^{\prime}}\right], \quad\left(\tilde{\alpha}_{0}\right)^{M}=\frac{G^{M N}}{\sqrt{2}}\left[n_{N}-\left(G_{N N^{\prime}}+B_{N N^{\prime}}\right) m^{N^{\prime}}\right] .
$$


In terms of the Kaluza-Klein and windings mode, the identification (2.12) enforced by the boundary state (for $n=0$ ) is independent of the closed string background and reads simply

$$
n_{M}=-2 \pi \alpha^{\prime}\left(F_{i}\right)_{M N} m^{N} .
$$

Let us end our brief comments on the string spectrum on magnetized tori by noticing that it is easy to generalize this analysis to all other configurations that are connected by T-duality to the case of magnetized D-branes. A generic T-duality transformation is encoded in a $O(d, d, Z)$ matrix

$$
T=\left(\begin{array}{cc}
a_{M}^{N} & b_{M N} \\
c^{M N} & d_{N}^{M}
\end{array}\right), \quad \text { with } \quad{ }^{\mathrm{t}} c a+{ }^{\mathrm{t}} a c={ }^{\mathrm{t}} b d+{ }^{\mathrm{t}} d b=0 \quad, \quad{ }^{\mathrm{t}} a d+{ }^{\mathrm{t}} c b=1 .
$$

where $a, b, c$ and $d$ are $2 d \times 2 d$ matrices. The matrix $T$ acts on the combination $G+B$ as follows (see [24] for a review 4 )

$$
\left(G^{\prime}+B^{\prime}\right)=[a(G+B)+b][c(G+B)+d]^{-1} .
$$

The same T-duality transformation is realized at the level of oscillators by multiplying the left and right-moving modes by specific matrices: $\alpha_{n} \equiv T_{+} \alpha_{n}^{\prime}, \tilde{\alpha} \equiv T_{-} \tilde{\alpha}_{n}^{\prime}$, where the primed oscillators are those obtained after the T-duality and

$$
\left(T_{+}\right)_{N}^{M}=\left\{[d+c(G+B)]^{-1}\right\}_{N}^{M}, \quad\left(T_{-}\right)_{N}^{M}=\left\{\left[d-c^{\mathrm{t}}(G+B)\right]^{-1}\right\}_{N}^{M} .
$$

Notice that by using (2.15) one can prove that ${ }^{t} T_{ \pm} G T_{ \pm}=G^{\prime}$. From (2.2) we immediately see that upon T-duality the reflection matrices change in a very simple way $R_{i} \rightarrow R_{i}^{\prime}=T_{-}^{-1} R_{i} T_{+}$. This implies that the monodromy matrices $R_{l k}$ change by a similarity transformation and that the twists $\epsilon_{l k}$ are invariant under T-duality. In particular, we can easily describe within the formalism of reflection and monodromy matrices also D-branes at angles. This situation is characterized by symmetric reflections matrices that square to one. If all $R_{i}$ 's can be brought in this form by means of the same T-duality transformation $T$, then it means that the D-brane setup can be geometrized completely and described in terms of D-branes at angles.

\section{Twisted partition function on $T^{2 d}$}

\subsection{Twisted partition function in the closed string channel}

The vacuum diagram we will compute has $g+1$ borders on different D-branes and no handles or crosscaps. In order to avoid the subtleties of the case with multiple wrappings [29], we will take all $l_{i M}$ 's in (2.1) to be equal one. Moreover we will take a second

\footnotetext{
${ }^{4}$ One has to take into account that in our conventions $\alpha_{n}$ and $\tilde{\alpha}_{n}$ are exchanged with respect to 24
} 
simplifying hypothesis and focus on the case of commuting fluxes. In formulae, our setup will satisfy

$$
l_{i M}=1, \quad \text { and } \quad\left[G^{-1} \mathcal{F}_{i}, G^{-1} \mathcal{F}_{j}\right]=0, \quad \forall i, j=0, \ldots, g .
$$

where $G$ and $\mathcal{F}_{i}$ are viewed as space-time matrices. Commutativity implies that all reflection matrices $R_{i}$ can be diagonalized simultaneously and so the eigenvalues $\epsilon_{l k}^{a}$ of the monodromy matrices $R_{l k}$ are simply $\epsilon_{l k}^{a}=e_{k}^{a}-e_{l}^{a}, \quad a=1,2, \ldots, d$, where $\mathrm{e}^{2 \pi \mathrm{i} e_{i}^{a}}$ are the eigenvalues of $R_{i}$.

The computation of this partition function in the closed string channel can be performed by using the boundary states formalism. This approach was used in [17] for the case of unmagnetized D-branes and here we will follow the same steps, the main difference being that in the present case the reflection matrices $R_{i}$ contain generic phases and not just \pm 1 . If we start from an off-shell closed string vertex in the $z$ coordinates (A.4) describing the emission of $g+1$ states and insert a first boundary state, then we get a disk parametrized as the upper half complex plane, where the real axis represents the boundary we have just inserted. On the contrary if we had worked in the $w$ coordinates (2.11), we would have obtained the disk of unit radius. Let us focus on the $z$ coordinates and saturate the remaining $g$ off-shell states with other boundary states. Each insertion cuts out a small disk in the upper half complex plane plus an image in the lower part and the circle and its image are identified. These non overlapping disks are completely specified by the $2 \times 2$ matrices

$$
S_{\mu}^{\mathrm{cl}}=\left(\begin{array}{cc}
a_{\mu}^{\mathrm{cl}} & b_{\mu}^{\mathrm{cl}} \\
c_{\mu}^{\mathrm{cl}} & d_{\mu}^{\mathrm{cl}}
\end{array}\right) \equiv \frac{1}{2 \sqrt{q_{\mu}}\left(\chi_{\mu}-\bar{\chi}_{\mu}\right)}\left(\begin{array}{cc}
\chi_{\mu}-q_{\mu} \bar{\chi}_{\mu} & -\left|\chi_{\mu}\right|^{2}\left(1-q_{\mu}\right) \\
\left(1-q_{\mu}\right) & q \chi_{\mu}-\bar{\chi}_{\mu}
\end{array}\right),
$$

with $\mu=1, \ldots, g$. The centers of the disks are in $a_{\mu} / c_{\mu}$ (and $-d_{\mu} / c_{\mu}$ for the images) and the radii (common to the disks and their images) are $\sqrt{q_{\mu}}\left|\bar{\chi}_{\mu}-\chi_{\mu}\right| /\left(1-q_{\mu}\right)$. Thus we get a world-sheet parameterization as in Fig. 2b, with $g+1$ borders which are the real axis plus the $g$ circles specified above. The (real) multipliers $q_{\mu}$ 's and the (complex) fixed points $\chi_{\mu}$ 's of Eq. (3.2) yield $3 g$ real parameters; as usual, three real parameters among the $\chi_{\mu}$ 's can be fixed arbitrarily thanks to the $S L(2, R)$ invariance, that in this context is simply the freedom to change all $S_{\mu}^{\mathrm{cl}}$ by a similarity transformation with a $S L(2, R)$ matrix. So we get the correct dimension $(3 g-3)$ for a the moduli space of a disk with $g+1$ boundaries. Notice that the parameters $q_{\mu}$ are directly related to the eigenvalues of the $S_{\mu}^{\mathrm{cl}}$ s (which are $\left\{\sqrt{q_{\mu}}, 1 / \sqrt{q_{\mu}}\right\}$ ) and so cannot be fixed by exploiting the $S L(2, R)$ invariance. The infinite group freely generated by the $g$ matrices (3.2) is called Schottky group (for a disk with $g+1$ boundaries). A generic element will be indicated by $T_{\alpha}^{\mathrm{cl}}$ and has the same form of the generators (3.2), so for each $T_{\alpha}^{\mathrm{cl}}$ we can derive a real $q_{\alpha}$ and a complex $\chi_{\alpha}$, which are calculable functions of the moduli $q_{\mu}, \chi_{\mu}$. In the operator formalism the interaction vertices are written in terms of a simple representation of the 


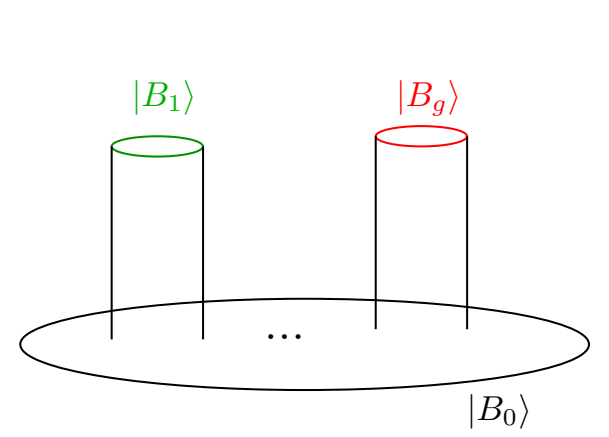

2

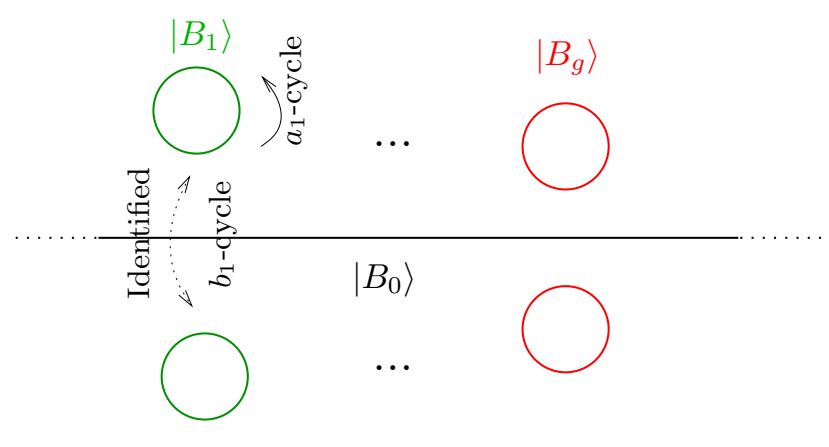

20

Figure 2: 2a represents the partition function under study from a space-time point of view; $2 \mathrm{~b}$ is a representation from the world-sheet point of view: the world-sheet is the upper half part of the complex plane that is outside all disks.

Schottky group [15]. Sewing together vertices and boundary states amounts to multiply together representations of Schottky elements, so it comes to no surprise that the final results for the partition function is given in terms of sums and products over the Schottky group. In the case of interest for us, the sewing procedure carry also a dependence on the space-time matrices $R_{i}$ contained in the boundary states. Here we will recall only the main features of the computation and the final result, while we leave to the Appendix $\mathrm{A}$ the discussion of some technical steps.

For the non-zero mode contributions $\left(\alpha_{n}\right.$ and $\tilde{\alpha}_{n}$, with $\left.n \neq 0\right)$ there is a nice pairing throughout the computation between the Schottky matrices $S_{\mu}^{\mathrm{cl}}$ and the spacetime matrices $\mathcal{S}_{\mu} \equiv R_{0 \mu}$ : each $\left(S_{\mu}^{\mathrm{cl}}\right)^{ \pm 1}$ is accompanied by the corresponding $\mathcal{S}_{\mu}^{ \pm 1}$. In our case all $\mathcal{S}_{\mu}$ 's can be diagonalized simultaneously, thus the non-zero mode contribution to vacuum diagram can be written in terms of the eigenvalues of $T_{\alpha}^{\mathrm{cl}}$ and those of $\mathcal{S}_{\mu}$. The first ones are basically the multipliers $q_{\alpha}$, while the second ones are just $\mathrm{e}^{ \pm 2 \pi \mathrm{i} \epsilon_{\mu}^{a}}$, with $\epsilon_{\mu}^{a} \equiv \epsilon_{0 \mu}^{a}=e_{\mu}^{a}-e_{0}^{a}$, for $\mu=1,2, \ldots, g$. Notice that from the world-sheet point of view the eigenvalues of $\mathcal{S}_{\mu}^{ \pm 1}$ are simply parameters that twist the periodicity conditions of the string coordinates along the $b^{\mathrm{cl}}$-cycles, that are those connecting each circle in Fig. 2 with its own image. This twist is easily tractable within the operator formalism [30, 18]. In formulae, the integrand for the twisted partition function can be written in terms of the $g$-loop untwisted result (all $F_{i}=0$ ) times $d$ factors of $\left[\mathcal{R}^{a}\left(\bar{\epsilon}^{a}\right)\right]_{g}^{\text {cl }}$ defined as follows

$$
\left[\mathcal{R}^{a}\left(\bar{\epsilon}^{a}\right)\right]_{g}^{\mathrm{cl}}=\frac{\prod_{\alpha}{ }^{\prime} \prod_{n=1}^{\infty}\left(1-q_{\alpha}^{n}\right)^{2}}{\prod_{\alpha}{ }^{\prime} \prod_{n=1}^{\infty}\left(1-\mathrm{e}^{-2 \pi \mathrm{i} \mathrm{\epsilon}^{a} \cdot \vec{N}_{\alpha}} q_{\alpha}^{n}\right)\left(1-\mathrm{e}^{2 \pi \mathrm{i} \vec{\epsilon}^{a} \cdot \vec{N}_{\alpha}} q_{\alpha}^{n}\right)} .
$$

In the vector $\vec{\epsilon}^{a}$ we have collected the $\epsilon_{\mu}^{a}, \mu=1,2, \ldots, g$; also $\vec{N}_{\alpha}$ is a vector with $g$ integer entries: the $\mu^{\text {th }}$ entry counts how many times the Schottky generator $S_{\mu}^{\mathrm{cl}}$ enters in the 
element of the Schottky group $T_{\alpha}^{\mathrm{cl}}$, whose multiplier is $q_{\alpha}$ (for example $S_{\mu}^{\mathrm{cl}}$ contributes 1 , while $\left(S_{\mu}^{\mathrm{cl}}\right)^{-1}$ contributes -1$)$. The primed product over the Schottky group means product over primary classes, that is one has to take only the elements that cannot be written as power of another element of the group; moreover one has to take only one representative for each conjugacy class (class of elements that are related by cyclic permutation of their constituents factors). In the non-compact case, the oscillator modes provide the only non-trivial contribution to the twisted partition function [30]

$$
\left.Z_{g}^{\mathrm{cl}}(F)\right|_{\text {uncomp. }}=\left(\prod_{i=0}^{g} \sqrt{\operatorname{Det}\left(1-G^{-1} \mathcal{F}_{i}\right)}\right) \int[d Z]_{g}^{\mathrm{cl}} \prod_{a=1}^{d}\left[\mathcal{R}^{a}\left(\bar{\epsilon}^{a}\right)\right]_{g}^{\mathrm{cl}}
$$

where $[d Z]_{g}^{\mathrm{cl}}$ is the integrand of the usual $\left(F_{i}=0\right)$ partition function in Minkowski space and the superscript $c l$ is just to recall that we are working in the closed string channel. Notice that the overall coefficient is just the usual Born-Infeld Lagrangian rescaled by a factor of $\sqrt{G}$, since all $F_{i}$-independent normalizations are included in $[d Z]_{g}^{\mathrm{cl}}$. Eq. (3.4) provides a direct generalization of the 1-loop result [31] to the multiloop case. In fact in this case there is only one conjugacy class and the product $\prod_{\alpha}{ }^{\prime}$ is absent. So (3.3) reduces to

$$
\left[\mathcal{R}^{a}\left(\epsilon^{a}\right)\right]_{g=1}^{\mathrm{cl}}=-2 \sin \left(\pi \epsilon^{a}\right) \frac{\eta^{3}\left(\tau^{\mathrm{cl}}\right)}{\theta_{11}\left(\epsilon^{a}, \tau^{\mathrm{cl}}\right)}
$$

where $\eta$ and $\theta_{11}$ are the usual Dedekind and odd Theta function with $q=\mathrm{e}^{2 \pi \mathrm{i} \tau^{\mathrm{cl}}}$.

Let us now consider the zero-mode contributions that are present in toroidal compactification. Each boundary state $\left|B_{i}\right\rangle$ contains a Kronecker delta selecting a particular combination of winding and Kaluza-Klein modes that couples to the D-brane, see Eq. (2.14). The closed string interaction vertex (A.4) contains two independent Kronecker delta's over winding and Kaluza-Klein modes. Thus the zero-mode contribution arises only from those modes that satisfy simultaneously all these conditions. By expressing all $\tilde{\alpha}_{0}$ in terms of $\alpha_{0}$, one can check that the zero-modes must satisfy the following condition:

$$
\sum_{\mu=1}^{g}\left(1-\mathcal{S}_{\mu}\right) \alpha_{0}^{\mu}=0
$$

In a long but straightforward computation one can follow the zero-mode contributions in the computation where the closed string vertex (A.4) is saturated with the boundary states. This basically amounts to follow what was done in the Appendix D of [15] and take into account the modifications induced by the space-time matrices $\mathcal{S}_{\mu}$. The tricky point is that the pairing between these $\mathcal{S}_{\mu}$ and the Schottky generators $S_{\mu}^{\mathrm{cl}}$ is spoiled in this part of the computation. This is due to (A.3) which implies that the matrices $D_{n m}$ appearing in the vertex and in the boundary states do not provide a true representation of 
the Schottky group in the zero-mode sector. In the Appendix $\mathrm{A}$ we provide some details of this computation, here let us give the final result for the zero-mode sector written in terms of the eigenvalues $\alpha_{0}$ of the left-moving momentum

$$
\exp \left\{\frac{1}{2} \sum_{\mu, \nu=1}^{g} \alpha_{0}^{\mu} G C_{\mu \nu}^{(1)} \alpha_{0}^{\nu}\right\}=\exp \left\{\frac{1}{2} \sum_{\mu, \nu=1}^{g} \alpha_{0}^{\mu} G\left[\mathcal{S}_{\mu}^{-1} \int_{w}^{S_{\mu}^{\mathrm{cl}}(w)} \boldsymbol{\zeta}_{\nu}(z) d z\right] \alpha_{0}^{\nu}\right\} .
$$

The $\boldsymbol{\zeta}_{\nu}(z)$ 's are Prym differential, i.e closed meromorphic 1-forms, periodic along the $a_{\mu}^{\mathrm{cl}}$ cycles and with fixed monodromies along the $b_{\mu}^{\mathrm{cl}}$-cycles (see Fig 2)

$$
\boldsymbol{\zeta}_{\nu}\left(S_{\mu}^{\mathrm{cl}}(z)\right) d\left(S_{\mu}^{\mathrm{cl}}(z)\right)=\mathcal{S}_{\mu} \boldsymbol{\zeta}_{\nu}(z) d z
$$

The sewing procedure gives an explicit expression for these 1-forms in terms of a sum over the Schottky group:

$$
\boldsymbol{\zeta}_{\nu}(z)=\sum_{\alpha} \mathcal{T}_{\alpha} \mathcal{S}_{\nu}\left[\frac{1}{z-T_{\alpha}^{\mathrm{cl}} S_{\nu}^{\mathrm{cl}}\left(z_{0}\right)}-\frac{1}{z-T_{\alpha}^{\mathrm{cl}}\left(z_{0}\right)}\right],
$$

where $\mathcal{T}_{\alpha}$ is the space-time counterpart of $T_{\alpha}$, with each $S_{\mu}^{\mathrm{cl}}$ 's replaced by a $\mathcal{S}_{\mu}$ 's. These Prym differentials were already found in [18] in the study of the twisted determinants for the Dirac operator, or in other words the partition function of a fermionic $b, c$ system of conformal weight $(1,0)$. The same pattern appears also in the untwisted case with the usual Abelian differentials (which are the limit of the $\boldsymbol{\zeta}_{\mu}(z)$ 's when all $\mathcal{S}_{\mu}=1$ ) and the reason is obviously that we are dealing with object of conformal weight 1 both in the case of the above mentioned $b, c$ system and in that of the string coordinates $\partial X$. RiemannRoch theorem implies that it is not possible to find $g$ such Prym differentials that are regular and in fact our definition depends on an arbitrary point $z_{0}$, where (3.9) has a simple pole. However it is not difficult to check that, thanks to the delta function (3.6), the exponent in (3.7) can be rewritten as

$$
\begin{array}{r}
\{\ldots\}=\frac{1}{2} \sum_{\hat{\mu}, \hat{\nu}=1}^{g-1} \alpha_{0}^{\hat{\mu}} G \mathcal{S}_{\hat{\mu}}^{-1}\left[\int_{w}^{S_{\hat{\mu}}^{\mathrm{cl}}(w)} \Omega_{\hat{\nu}}(z)-\frac{1-\mathcal{S}_{\hat{\mu}}}{1-\mathcal{S}_{g}} \int_{w}^{S_{g}^{\mathrm{cl}}(w)} \boldsymbol{\Omega}_{\hat{\nu}}(z)\right] \alpha_{0}^{\hat{\nu}} \\
\text { where } \boldsymbol{\Omega}_{\hat{\nu}}(z)=\boldsymbol{\zeta}_{\hat{\nu}}(z)-\frac{1-\mathcal{S}_{\hat{\nu}}}{1-\mathcal{S}_{g}} \boldsymbol{\zeta}_{g}(z), \text { with } \hat{\nu}=1, \ldots, g-1,
\end{array}
$$

where we have supposed $\mathcal{S}_{g} \neq 1$; we need not to worry about the ordering of the matrices $\mathcal{S}_{\mu}$ since they all commute. In ref. [18] it is shown that in (3.10) the dependence on $z_{0}$ disappears 5 . Therefore the $\boldsymbol{\Omega}_{\hat{\nu}}(z)$ 's are a set of $g-1$ regular Prym differential and

\footnotetext{
${ }^{5}$ This is also evident from (3.15).
} 
provide a complete basis for this space. This is the basis that is naturally selected by the sewing procedure. It is also possible to check that (3.10) does not depend on the the curve representing the $b_{\hat{\mu}}^{\mathrm{cl}}$-cycles, i.e. connecting $w$ and $S_{\hat{\mu}}^{\mathrm{cl}}(w)$. First, $\boldsymbol{\Omega}_{\hat{\nu}}(z) d z$ is a closed and regular differential so the exact form of the path is immaterial. Then we can check the independence of the point $w$, by taking the derivative of the exponent with respect to $w$ and showing that it is zero. This is more easily done by using the form (3.7). Thanks to the periodicities (3.8). we get

$$
\frac{d}{d w}\{\ldots\}=\left\{\frac{1}{2} \sum_{\mu, \nu=1}^{g} \alpha_{0}^{\mu} G\left(1-\mathcal{S}_{\mu}^{-1}\right) \boldsymbol{\zeta}_{\nu}(z) \alpha_{0}^{\nu}\right\},
$$

where the sums over $\mu$ and $\nu$ are decoupled. Then by using (2.5) and the presence of the constraint (3.6) in the integrand one can see that (3.12) vanishes implying that the exponential contribution due to the zero-modes is independent of $w$. The independence of (3.10) of $w$ means that the integrals span a closed contour on the branched Riemann surface. In terms of the basic cycles of the closed string parameterization the integral in (3.10) forms the closed contour $b_{\hat{\mu}}^{\mathrm{cl}} b_{g}^{\mathrm{cl}}\left(b_{\hat{\mu}}^{\mathrm{cl}}\right)^{-1}\left(b_{g}^{\mathrm{cl}}\right)^{-1}$. We will depict this path in the open string parameterization, see Fig. 5 .

Thus we can put together the oscillators and the zero-mode contribution and write the full bosonic partition function of Fig. 2 for magnetized bosonic D-branes on a $T^{2 d}$

$$
\begin{aligned}
Z_{g}^{\mathrm{cl}}(F) & =\left(\prod_{i=0}^{g} \sqrt{\operatorname{Det}\left(1-G^{-1} \mathcal{F}_{i}\right)}\right) \int[d Z]_{g}^{\mathrm{c}} \prod_{a=1}^{d}\left[\mathcal{R}^{a}\left(\vec{\epsilon}^{a}\right)\right]_{g}^{\mathrm{cl}} \sum_{m} \delta\left[\left(1-\mathcal{S}_{\mu}\right) \alpha_{0}^{\mu}\right] \\
& \times \exp \left\{\pi \mathrm{i} \sum_{\hat{\mu}, \hat{\nu}=1}^{g-1} \alpha_{0}^{\hat{\mu}} G \mathcal{S}_{\hat{\mu}}^{-1 / 2} \boldsymbol{D}_{\hat{\mu} \hat{\nu}} \mathcal{S}_{\hat{\nu}}^{1 / 2} \alpha_{0}^{\hat{\nu}}\right\},
\end{aligned}
$$

where the $\alpha_{0}$ 's are functions of the windings $m$ as dictated by Eqs. (2.13) and (2.14) and

$$
\boldsymbol{D}_{\hat{\mu} \hat{\nu}}=\frac{1}{2 \pi \mathrm{i}} \mathcal{S}_{\hat{\mu}}^{-1 / 2}\left[\int_{w}^{S_{\hat{\mu}}^{\mathrm{cl}}(w)}-\frac{1-\mathcal{S}_{\hat{\mu}}}{1-\mathcal{S}_{g}} \int_{w}^{S_{g}^{\mathrm{cl}}(w)}\right] \boldsymbol{\Omega}_{\hat{\nu}}(z) d z \mathcal{S}_{\hat{\nu}}^{-1 / 2}, \quad \hat{\mu}, \hat{\nu}=1,2, . ., g-1 .
$$

In the special case of a two-dimensional torus, $T^{2}$, our (3.10) is directly connected to the results obtained in [12]. Let us see give some more details to see how the connection between the two formulations works.

The 1 -forms $\boldsymbol{\zeta}_{\nu}(z) d z$ and $\boldsymbol{\Omega}_{\hat{\nu}}(z) d z$ display a space-time dependence that can be more easily expressed in the basis where all the monodromy matrices $R_{\mu}$ are diagonal. In this eigenvector basis also $\zeta_{\nu}$ and $\Omega_{\hat{\nu}}$ are diagonal and each entry $\zeta_{\nu}^{ \pm \epsilon^{a}}$ or $\Omega_{\hat{\nu}}^{ \pm \vec{\epsilon}^{a}}$ depends on the eigenvalues $\mathrm{e}^{ \pm 2 \pi \mathrm{i} \bar{\epsilon}^{a}}$ of the corresponding eigenvector. The $\Omega_{\hat{\nu}}^{ \pm \bar{\epsilon}^{a}} d z$ are closely related to 
the 1-forms $\omega$ 's of [12]. In fact one can see that their $\omega_{\hat{\mu}}$ corresponds to our combination $\mathrm{e}^{-\pi \mathrm{i} \epsilon_{\hat{\mu}}} \Omega_{\hat{\mu}}^{\epsilon^{u}} /(2 \pi \mathrm{i})$. In particular one can recover the approximated expression (A.35) of 12 by rewriting the Prym differential to be inserted in (3.11) in the following form [18]6]

$$
\begin{aligned}
\zeta_{\nu}^{\vec{\epsilon}^{a}}(z) & =\sum_{\alpha}^{(\nu)} \mathrm{e}^{2 \pi \mathrm{i}\left(\bar{\epsilon}^{a} \cdot \vec{N}_{\alpha}+\epsilon_{\nu}^{a}\right)}\left[\frac{1}{z-T_{\alpha}^{\mathrm{cl}}\left(\chi_{\nu}\right)}-\frac{1}{z-T_{\alpha}^{\mathrm{cl}}\left(\bar{\chi}_{\nu}\right)}\right] \\
& +\left(1-\mathrm{e}^{2 \pi \mathrm{i} \epsilon_{\nu}^{a}}\right) \sum_{\alpha} \mathrm{e}^{2 \pi \mathrm{i}^{a} \cdot \vec{N}_{\alpha}}\left[\frac{1}{z-T_{\alpha}^{\mathrm{cl}}\left(z_{0}\right)}-\frac{1}{z-T_{\alpha}^{\mathrm{cl}}\left(a_{\nu}^{\alpha}\right)}\right],
\end{aligned}
$$

and by taking in the sums only the contribution of $T_{\alpha}^{\mathrm{cl}}=1$.

In [12], the zero-mode contribution to the twisted partition function is computed in a IIA setup where D-branes intersect on a $T^{2}$. By performing a T-duality we can rewrite the exponential term in (3.13) in this language. On a $T^{2}$ we can write the metric and the $B$ field in the integral basis as follows

$$
G=\frac{T_{2}}{U_{2}}\left(\begin{array}{cc}
1 & U_{1} \\
U_{1} & |U|^{2}
\end{array}\right) \quad \text { and } \quad B=\left(\begin{array}{cc}
0 & -T_{1} \\
T_{1} & 0
\end{array}\right)
$$

where $T$ and $U$ are the Kähler and complex structure of the torus. This parameterization is very convenient in the discussion of T-duality transformations. Indeed, a T-duality along the $x=x^{1}$ axis amounts just to the exchange $T \leftrightarrow U$, while a T-duality along $y=x^{2}$ corresponds to $T \leftrightarrow-1 / U$. For our computation, we will need two other matrices:

$$
2 \pi \alpha^{\prime} F_{i}=\left(\begin{array}{cc}
0 & f_{i} \\
-f_{i} & 0
\end{array}\right), \quad \text { and } \mathcal{E}=\sqrt{\frac{T_{2}}{2 U_{2}}}\left(\begin{array}{ll}
1 & U \\
1 & \bar{U}
\end{array}\right)
$$

where the magnetic fields $f_{i}$ 's are integers as consequence of (2.1) and (3.1), and $\mathcal{E}$ is the Vielbein matrix transforming the real integral coordinates into the complex ones that diagonalize the $R_{i}$ 's. In the complex basis each element of $\boldsymbol{D}$ (defined in (3.14)) is a diagonal space-time matrix. By comparing our conventions and those of [12] we see that its first component $D_{\hat{\mu} \hat{\nu}}(\epsilon)$ agrees with the definition given in the second line of (A.16) of [12. The second component has, of course, opposite twists and will be indicated by $D_{\hat{\mu} \hat{\nu}}(-\epsilon)$. If we use (2.13) and (2.14), we can re-express the left momentum $\alpha_{0}^{i}$ in terms of the integer windings

$$
\mathcal{E} \alpha_{0}^{i}=\frac{1}{\sqrt{2}} \mathcal{E} G^{-1}\left(G-B-2 \pi \alpha^{\prime} F_{i}\right) m_{i}=\frac{\left|v_{i}\right|}{\sqrt{2}} R_{0}^{\prime-1 / 2} \mathcal{S}_{i}^{\prime}{ }^{-1 / 2} \frac{|U|}{\sqrt{T_{2} U_{2}}} \mathcal{E} m_{i}, \quad i=0,1, \ldots g,
$$

\footnotetext{
${ }^{6}$ In the first line, $\sum_{\alpha}{ }^{(\nu)}$ means that in $T_{\alpha}^{\mathrm{cl}}$ the last factor on the right cannot be a positive or negative power of $S_{\nu}^{\mathrm{cl}}$; in the second line, $a_{\nu}^{\alpha}=\chi_{\nu}$ if $T_{\alpha}^{\mathrm{cl}}$ is of the form $T_{\alpha}^{\mathrm{cl}}=T_{\beta}^{\mathrm{cl}}\left(S_{\nu}^{\mathrm{cl}}\right)^{l}$ with $l \geq 1$, while $a_{\nu}^{\alpha}=\bar{\chi}_{\nu}$ otherwise.
} 


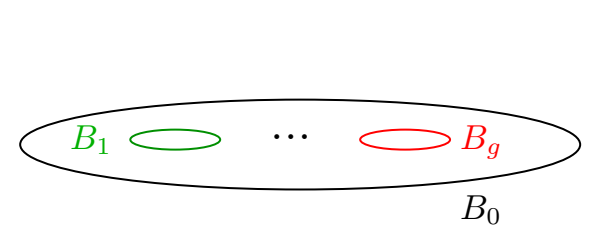

[3.

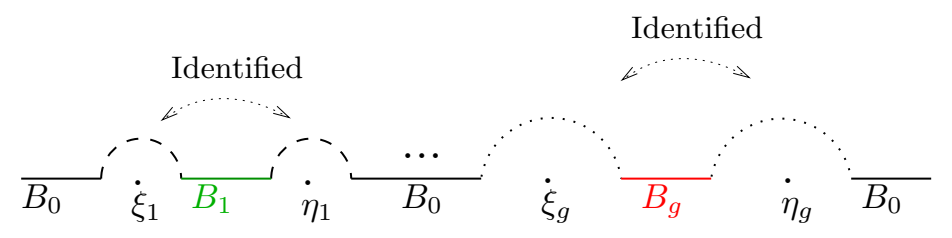

\$b

Figure 3: $3 \mathrm{a}$ is a space-time representation of the same partition function of Fig. 2 in the open string channel; $3 \mathrm{~b}$ is the corresponding world-sheet surface in the Schottky parametrization.

where the primed matrices are written in the complex basis, where they are diagonal, $m_{i}$ is a column vector with the windings $m_{i}^{1}, m_{i}^{2}$, and $\left|v_{i}\right|$ is the T-dual (along $y$ ) of the same quantity appearing in [12]

$$
\left|v_{i}\right|=\sqrt{\frac{U_{2}}{|U|^{2}} \frac{|T|^{2}}{T_{2}}} \frac{\left|\mathrm{i}\left(f_{i}-T_{1}\right)+T_{2}\right|}{|T|} .
$$

By using (3.14) and (3.18) into (3.13) for the $T^{2}$ configuration under study we see that the exponent in (3.13) can be rewritten as

$$
\frac{\pi \mathrm{i}}{4}\left(m_{\hat{\mu}}^{1}+\bar{U} m_{\hat{\mu}}^{2}\right)\left[D_{\hat{\mu} \hat{\nu}}(\epsilon)+D_{\hat{\nu} \hat{\mu}}(-\epsilon)\right]\left(m_{\hat{\nu}}^{1}+U m_{\hat{\nu}}^{2}\right) \frac{|U|^{2}}{U_{2}^{2}}\left|v_{\hat{\mu}}\right|\left|v_{\hat{\nu}}\right| .
$$

By using the properties of $D( \pm \epsilon)$ derived in Eqs. (A.14, A.15, A.18) of [12, one can check that $D(\epsilon)+{ }^{\mathrm{t}} D(-\epsilon)$ coincides with the double of the matrix $\tau$ defined in that paper and that, after a T-duality $U \leftrightarrow-1 / T$, (3.20) agrees with (5.18) of [12], apart from an overall factor of $1 / 2$. By using the same equations of [12], one can also get the interesting identity:

$$
D_{\hat{\mu} \hat{\nu}}(\epsilon)-D_{\hat{\nu} \hat{\mu}}(-\epsilon)=2 \mathrm{i} \frac{\sin \left(\pi \epsilon_{\hat{\mu}}\right) \sin \left(\pi \epsilon_{\hat{\nu}}-\pi \epsilon_{g}\right)}{\sin \left(\pi \epsilon_{g}\right)}, \text { for } \hat{\nu} \geq \hat{\mu},
$$

that will be checked in Section 4.2 for $g=2$, in the degeneration limit of the open string channel.

\subsection{Twisted partition function in the open string channel}

The aim of this section is to perform a modular transformation and to rewrite the result (3.13) in the open string channel. Pictorially this map transforms the world-sheet of Fig. 2 into the one of Fig. 3. Again the Schottky parameterization of the world-sheet in 
Fig. 3b is completely specified in terms of $g 2 \times 2$ matrices 7

$$
S_{\mu}=\left(\begin{array}{cc}
a_{\mu} & b_{\mu} \\
c_{\mu} & d_{\mu}
\end{array}\right) \equiv \frac{1}{\sqrt{k_{\mu}}\left(\eta_{\mu}-\xi_{\mu}\right)}\left(\begin{array}{cc}
\eta_{\mu}-k_{\mu} \xi_{\mu} & -\eta_{\mu} \xi_{\mu}\left(1-k_{\mu}\right) \\
\left(1-k_{\mu}\right) & k_{\mu} \eta_{\mu}-\xi_{\mu}
\end{array}\right)
$$

where now the fixed points $\eta, \xi$ (which are the analogue of $\chi, \bar{\chi}$ of (3.2)) are all real. The open string world-sheet is the upper half part of the complex plane that is outside all the circles defined by the $S_{\mu}$ 's as explained after Eq. (3.2). These circles are identified pairwise (see Fig. 3b).

As usual, the modular transformation we are interested in is non-analytic in the Schottky parameters. This is already manifest in the one loop case where we have $\ln q=4 \pi^{2} / \ln k$. In order to circumvent this technical problem we follow the approach used in [19, 25] for the twisted partition function in Minkowski space. We first rewrite the products over the Schottky group in terms of genus $g$ Theta functions and other geometrical object such as the Prime Form. This can be done thanks to the identities derived in [18] which are consequence of the equivalence between fermionic and bosonic theories in two dimensions. In this description we can perform explicitly the modular transformation that exchanges the $a$ and the $b$-cycles 8 as follows: $a_{\mu}^{\mathrm{cl}}=b_{\mu}$ and $b_{\mu}^{\mathrm{cl}}=a_{\mu}^{-1}$. After this map we have a world-sheet surface that looks like Fig. 3a. For computational convenience we then use once more the identities of [18] to rewrite the result in terms of the open string Schottky groups (generated by the $S_{\mu}$ 's in (3.22)). In the non-compact case, this computation was performed in [19, 25] and the result for the partition function in the open string channel is

$$
\left.Z_{g}(F)\right|_{\text {uncomp. }}=\left[\prod_{i=0}^{g} \sqrt{\operatorname{Det}\left(1-G^{-1} \mathcal{F}_{i}\right)}\right] \int[d Z]_{g} \prod_{a=1}^{d}\left[\mathrm{e}^{-\mathrm{i} \pi \vec{\epsilon}^{\vec{a}} \cdot \tau \cdot \vec{\epsilon}^{a}} \frac{\operatorname{det}(\tau)}{\operatorname{det}\left(T_{\bar{\epsilon}^{a}}\right)} \mathcal{R}_{g}\left(\bar{\epsilon}^{a} \cdot \tau\right)\right]
$$

Here $\tau_{\mu \nu}$ is the usual period matrix (written in the open string channel) and det is the determinant over the "loop" indices $\mu, \nu=1,2, \ldots, g ; T_{\vec{\epsilon}^{a}}$ is a twisted generalization of the period matrix

$$
\begin{gathered}
\left(T_{\vec{\epsilon}^{a}}\right)_{\hat{\nu} \hat{\mu}}=\frac{1}{2 \pi \mathrm{i}} \int_{w}^{S_{\hat{\nu}}(w)} \widehat{\Omega}_{\hat{\mu}}^{\vec{\epsilon}^{a}}(z) d z \text {, where } \widehat{\Omega}_{\hat{\mu}}^{\bar{\epsilon}^{a}}(z)=\left[\Omega_{\hat{\mu}}^{\vec{\epsilon}^{a} \cdot \tau}(z) \mathrm{e}^{\frac{2 \pi \mathrm{i}}{g-1} \bar{\epsilon}^{a} \cdot \vec{\Delta}_{z}}\right] \\
\left(T_{\vec{\epsilon}^{a}}\right)_{\hat{\nu} g}=\left(T_{\vec{\epsilon}^{a}}\right)_{g \hat{\mu}}=0 \quad, \quad \text { and }\left(T_{\vec{\epsilon}^{a}}\right)_{g g}=\frac{\mathrm{e}^{2 \pi \mathrm{i}\left(\bar{\epsilon}^{a} \cdot \tau\right) g}-1}{\mathrm{e}^{2 \pi \mathrm{e}_{g}^{a}}-1} .
\end{gathered}
$$

\footnotetext{
${ }^{7}$ We have endowed the quantities in the closed string channel with a suffix "cl"; those without any suffix will refer to the open string channel, if not otherwise specified.

${ }^{8}$ In the closed string parametrization, Fig. 20, we define the $b_{\mu}^{\mathrm{cl}}$-cycles to be the segments between $w$ and $S_{\mu}^{\mathrm{cl}}(w)$, while the $a_{\mu}^{\mathrm{cl}}$-cycles are the contours around the repulsive fixed points $\bar{\chi}_{\mu}$ clockwise oriented. In a similar way, in the open string parametrization, the $b$-cycles are the segments between $w$ and $S_{\mu}(w)$, while the $a$-cycles are the contours around the $\xi_{\mu}$ 's clockwise oriented.
} 
We will also use $\widetilde{T}_{\vec{\epsilon}^{a}}$ to indicate the $(g-1) \times(g-1)$ block of $T_{\vec{\epsilon}^{a}}$ which has indices $\hat{\mu}, \hat{\nu}=1, \ldots, g-1$. The Prym differentials $\Omega_{\mu}^{\vec{e}^{a} \cdot \tau}$ in this equation have the same functional form of those in (3.11), but with the following changes:

$$
\bar{\epsilon}^{a} \rightarrow \bar{\epsilon}^{a} \cdot \tau, \quad(\bar{\chi}, \chi) \rightarrow(\xi, \eta), \quad S_{\mu}^{\mathrm{cl}} \rightarrow S_{\mu} .
$$

$\vec{\Delta}_{z}$ is the vector of Riemann constants (or Riemann class) defined with respect to the base point $z$. When we change the base point, $\vec{\Delta}$ changes in a simple way determined by the usual Abelian differentials $\omega$

$$
\vec{\Delta}_{(z)}=\vec{\Delta}_{\left(z_{0}\right)}-\frac{g-1}{2 \pi \mathrm{i}} \int_{z_{0}}^{z} \vec{\omega}
$$

Then it is easy to see that the differentials $\widehat{\Omega}^{\epsilon^{a}}$ in (3.24) are twisted only along the $a_{\mu}$-cycles (of the open string channel) with phases $\mathrm{e}^{-2 \pi \mathrm{i} \epsilon_{\mu}^{a}}$. The identities necessary to rewrite (3.4) as (3.23) are Eqs. (13), (14) and (16) of [19]. They imply also that $\operatorname{det}\left(T_{\vec{\epsilon}^{a}}\right)$ is invariant under $\bar{\epsilon}^{a} \rightarrow-\bar{\epsilon}^{a}$ and can be used to derive the modular transformation of each single $\Omega_{\hat{\nu}}(z)$.

In fact to calculate $Z_{g}(F)$ in the compact case we need to express the matrix $\boldsymbol{D}_{\hat{\mu} \hat{\nu}}$ defined in (3.14) in terms of open string quantities. From [19] we get a relation between the determinants in the closed and open string parameterization and from there the modular transformation for each $\Omega_{\hat{\nu}}$ :

$$
\overline{\operatorname{det}}\left[\boldsymbol{\Omega}_{\hat{\nu}}\left(z_{\hat{\mu}}\right)\right]=\frac{1-\mathcal{S}_{g}^{\epsilon \cdot \tau}}{1-\mathcal{S}_{g}^{\epsilon}} \frac{\overline{\operatorname{det}}\left[\widehat{\boldsymbol{\Omega}}_{\hat{\nu}}\left(z_{\hat{\mu}}\right)\right]}{\operatorname{det} \boldsymbol{T}}=\frac{\overline{\operatorname{det}}\left[\widehat{\boldsymbol{\Omega}}_{\hat{\nu}}\left(z_{\hat{\mu}}\right)\right]}{\overline{\operatorname{det}} \widetilde{\boldsymbol{T}}},
$$

where $\widehat{\Omega}_{\hat{\nu}}, \quad \boldsymbol{T}_{\nu \mu}$ and $\widetilde{\boldsymbol{T}}_{\hat{\nu} \hat{\mu}}$ are $2 d \times 2 d$ matrices whose elements in the complex basis (where they are diagonal) are the $\widehat{\Omega}_{\hat{\nu}}^{ \pm \bar{\epsilon}^{a}},\left(T_{ \pm \vec{\epsilon}^{a}}\right)_{\nu \mu}$ and $\left(\widetilde{T}_{ \pm \vec{\epsilon}^{a}}\right)_{\hat{\nu} \hat{\mu}}$ defined in Eq. (3.24). The determinants $\overline{d e t}$ are taken only over the indices $\hat{\mu}, \hat{\nu}=1, \ldots, g-1$ and the superscripts for the matrices $\mathcal{S}_{g}$ remind that the eigenvalues are either $\epsilon_{g}$ or $(\epsilon \cdot \tau)_{g}$. We can single out each differential $\Omega_{\hat{\nu}}$ simply by integrating the l.h.s. of (3.27) on the variables $z_{\hat{\mu}}$ with $\hat{\mu} \neq \hat{\nu}$ along the $a_{\hat{\mu}}^{\mathrm{cl}}$-cycles and using the normalization property of the $\Omega_{\hat{\nu}}{ }^{\prime} \mathrm{s}: \oint_{a_{\hat{\mu}}^{\mathrm{cl}}} \Omega_{\hat{\nu}}=2 \pi \mathrm{i} \delta_{\hat{\mu} \hat{\nu}}$. The r.h.s. of (3.27) is thus integrated along the corresponding $b_{\hat{\mu}}$-cycles and we get

$$
\boldsymbol{\Omega}_{\hat{\nu}}(z)=\sum_{\hat{\rho}=1}^{g-1} \widehat{\boldsymbol{\Omega}}_{\hat{\rho}}(z) \widetilde{\boldsymbol{T}}_{\hat{\rho} \hat{\nu}}^{-1}, \quad \hat{\nu}=1,2, \ldots, g-1 .
$$

where the inverse of $\widetilde{\boldsymbol{T}}$ in the last step is over both the loop and the space-time indices. Notice that this is the natural generalization of the usual modular property of the Abelian differentials to the twisted case of Prym differentials, where the matrix $\widetilde{\boldsymbol{T}}$ plays the role of the period matrix. 
Now we can write the generalization of (3.23) to the case of toroidal compactification

$$
\begin{aligned}
Z_{g}(F) & =\left[\prod_{i=0}^{g} \sqrt{\operatorname{Det}\left(1-G^{-1} \mathcal{F}_{i}\right)}\right] \int[d Z]_{g} \sum_{m} \delta\left[\left(1-\mathcal{S}_{\mu}\right) \alpha_{0}^{\mu}\right] \\
& \times \exp \left\{\pi \mathrm{i} \sum_{\hat{\mu}, \hat{\nu}=1}^{g-1} \alpha_{0}^{\hat{\mu}} G \mathcal{S}_{\hat{\mu}}^{-1 / 2} \boldsymbol{D}_{\hat{\mu} \hat{\nu}} \mathcal{S}_{\hat{\nu}}^{1 / 2} \alpha_{0}^{\hat{\nu}}\right\} \prod_{a=1}^{d}\left[\mathrm{e}^{-\mathrm{i} \pi \vec{\epsilon}^{\vec{a}} \cdot \tau \cdot \bar{\epsilon}^{a}} \frac{\operatorname{det}(\tau)}{\operatorname{det}\left(T_{\bar{\epsilon}^{a}}\right)} \mathcal{R}_{g}\left(\vec{\epsilon}^{a} \cdot \tau\right)\right],
\end{aligned}
$$

where the matrix $\boldsymbol{D}$ of (3.14) is written in terms of the open string variables:

$$
\boldsymbol{D}_{\hat{\mu} \hat{\nu}}=\frac{1}{2 \pi \mathrm{i}} \mathcal{S}_{\hat{\mu}}^{-1 / 2}\left[\int_{a_{\hat{\mu}}^{-1}}-\frac{1-\mathcal{S}_{\hat{\mu}}}{1-\mathcal{S}_{g}} \int_{a_{g}^{-1}}\right] \boldsymbol{\Omega}_{\hat{\nu}}(z) d z \mathcal{S}_{\hat{\nu}}^{-1 / 2},
$$

and $\boldsymbol{\Omega}_{\hat{\nu}}$ is given in terms of the open string channel Prym differentials $\widehat{\Omega}_{\hat{\rho}}$ by Eq. (3.28).

The complication of this formula lies in the fact that it is not algebraic since, contrary to what happens in the untwisted case, there are still various integrals that we are not able to perform in general. On the other hand it is written in a form that is particular useful in the study of the degeneration limits as we will now see.

\section{Yukawa couplings from the partition function}

\subsection{The uncompact case}

For $g=2$, the Eq. (3.29) describes the twisted bosonic partition function depicted in Fig. 4. In the world-sheet parametrization, we choose to fix $\xi_{1}=\infty$ and $\eta_{1}=0$ for the

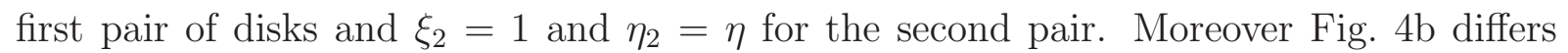
from Fig. $3 \mathrm{~b}$ with $g=2$ because we have changed the role of the repulsive and attractive points of the Schottky generator $S_{2}$, amounting to send $S_{2}$ into $S_{2}^{-1}$; this has been done to follow usual conventions for the open string, but has the price that from now on $\epsilon_{2}$ will be the opposite of the one used in the previous Sections.

We now will focus on the degeneration limit depicted in Fig. 1b that is captured by the following region of the world-sheet moduli space

$$
0<k_{1}<k_{2} \ll \eta \ll 1 .
$$

The formalism derived in the previous section is particularly suitable for studying quantitatively the limit (4.1), since in this approximation we need to keep only the first terms in all the Schottky series or products. The aim of this section is to focus on the uncompact case and compute explicitly the leading order contribution to (3.23) as function of the world-sheet moduli and the background fields. Then, as anticipated in the Introduction, 


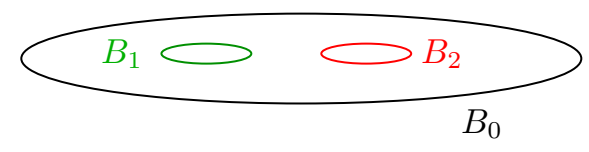

4.

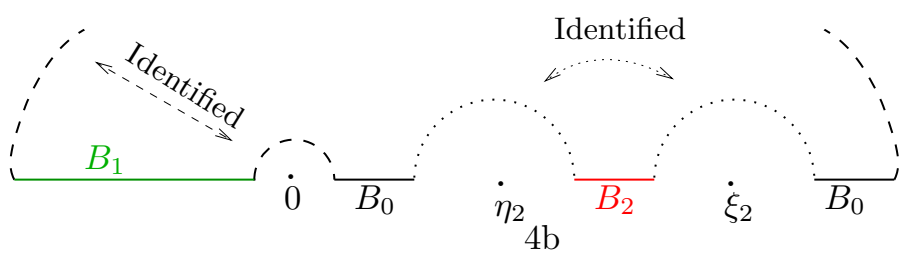

40

Figure 4: This figure represents the special case $g=2$ of the partition function depicted in Fig. 3. Notice that for this case we choose a slightly different the world-sheet parametrization (40) with the respect to the general case: we choose to have $\xi_{1}=\infty$ and $\eta_{1}=0$ and to swap the order of $\xi_{2}$ and $\eta_{2}$.

we will obtain by factorization the coupling among three twists fields. Notice that the limit (4.1) is similar to that studied in [25], where the twisted string partition function was connected to the field theory Euler-Heisenberg effective action induced by a charged scalar. The main difference is that now we do not take any scaling for the $\epsilon_{\mu}$ 's.

As a first step, let us write all the objects entering in (3.23) at the first orders in the limit (4.1). The Schottky product $\mathcal{R}_{2}(\epsilon \cdot \tau)$ is just one at the leading order, so we need to focus only on $T_{\vec{e}^{a}}$ and $\tau$

$$
\begin{aligned}
& \zeta_{1}^{\vec{\epsilon} \cdot \tau}(z) \simeq \frac{E_{1}}{z}+\left[\left(1-E_{1}\right)\left(\frac{1}{z-z_{0}}\right)+\left(1-E_{1}\right)\left(\frac{E_{1}}{z-k_{1} z_{0}}-\frac{E_{1}}{z}\right)\right], \\
& \zeta_{2}^{\vec{\epsilon} \cdot \tau}(z) \simeq \frac{E_{2}}{z-\eta}-\frac{E_{2}}{z-1}+\left(1-E_{2}\right)\left(\frac{1}{z-z_{0}}-\frac{1}{z-1}\right) \\
& +\left[\frac{E_{1} E_{2}}{z-k_{1} \eta}-\frac{E_{1} E_{2}}{z-k_{1}}+\left(1-E_{2}\right)\left(\frac{E_{1}}{z-k_{1} z_{0}}-\frac{E_{1}}{z-k_{1}}\right)\right] \text {, } \\
& E_{i}=\mathrm{e}^{2 \pi \mathrm{i}\left(\epsilon_{1} \tau_{1 i}+\epsilon_{2} \tau_{2 i}\right)}, \quad \tau \simeq \frac{1}{2 \pi \mathrm{i}}\left(\begin{array}{cc}
\ln k_{1} & \ln \eta \\
\ln \eta & \ln k_{2}
\end{array}\right), \\
& 2 \pi \mathrm{i} \vec{\Delta}_{z} \simeq\left\{-\frac{\ln k_{1}}{2}+\mathrm{i} \pi-\ln z,-\frac{\ln k_{2}}{2}+\mathrm{i} \pi+\ln \left(\frac{z-1}{z-\eta} \frac{z-k_{1}}{z-k_{1} \eta}\right)\right\} .
\end{aligned}
$$

The first line is obtained from (3.15) with the substitution (3.25), where we kept only the identity and $S_{1}$ in the Schottky sums since each term in the square parenthesis in (3.15) is of order $\prod_{\mu} k_{\mu}^{N_{\mu}}$. Actually at first sight the terms related to the element $S_{1}$, are subleading in the limit (4.1), but we need to keep them since they yield a finite contribution to the integral (3.24). After we reduced the Schottky series to the sum of these few terms, we are free to neglect also the terms that contain an explicit $z_{0}$ dependence, since we known that they do not contribute to $\Omega$. Now we need to evaluate the integral (3.24) in the limit (4.1), where the integrand is just a sum of five terms that have branch-cuts in $\left\{0, \eta k_{1}, k_{1}, \eta, 1\right\}$. We take the arbitrary point $w$ in (3.24) to coincide with $\eta$. With this 
choice all the integrals take the following form

$$
I(x) \equiv-\int_{k_{1} \eta}^{\eta} \frac{d z}{z-x}\left(\frac{z-1}{z-\eta} \frac{z-k_{1}}{z-k_{1} \eta}\right)^{\epsilon_{2}}\left(\frac{1}{z}\right)^{\epsilon_{1}}
$$

where $x=\left\{0, \eta k_{1}, k_{1}, \eta, 1\right\}$. Since $z \ll 1$ we can approximate $z-1$ just with -1 and after a change of variables that brings the region of integration to the interval $[0,1]$, the integral (4.3) coincides with the integral representation of the Appell Hypergeometric function $F_{1}$

$$
\int_{0}^{1} t^{a-1}(1-t)^{c-a-1}\left(1-t y_{1}\right)^{-b_{1}}\left(1-t y_{2}\right)^{-b_{2}} d t=\frac{\Gamma(a) \Gamma(c-a)}{\Gamma(c)} F_{1}\left(a ; b_{1}, b_{2} ; c ; y_{1}, y_{2}\right) .
$$

At this point we have to extract the leading order contribution in the $F_{1}$ 's. This is a somewhat delicate step because in the limit (4.1) both $y_{i}$ in (4.4) tend to one, so the standard series representation of $F_{1}$ is not useful. However the two $y_{i}$ 's scale in an ordered fashion. In fact from (4.3) we see that we can pose $y_{1}=\left(1-k_{1}\right) /\left(1-k_{1} / \eta\right)$ and $y_{2}=\left(1-k_{1}\right)$ so, in the limit (4.1), we have $\left|1-y_{2}\right| \ll\left|1-y_{1}\right|$. In this case we can use the following approximation

$$
\begin{aligned}
F_{1}\left(a ; b_{1}, b_{2} ; c ; y_{1}, y_{2}\right) & \sim \frac{B\left(c-b_{1}-b_{2}-a, a\right)}{B(a, c-a)}+\left(1-y_{1}\right)^{-b_{1}}\left(1-y_{2}\right)^{c-b_{2}-a} \frac{\Gamma(c) \Gamma\left(a+b_{2}-c\right)}{\Gamma(a) \Gamma\left(b_{2}\right)} \\
& +\left(1-y_{1}\right)^{c-b_{1}-b_{2}-a} \frac{\Gamma(c) \Gamma\left(c-b_{2}-a\right) \Gamma\left(b_{1}+b_{2}+a-c\right)}{\Gamma(a) \Gamma(c-a) \Gamma\left(b_{1}\right)}, \quad \text { (4.5) }
\end{aligned}
$$

where $B(a, b)$ is the Euler beta function. It is convenient to introduce the more symmetric variables [32]

$$
q_{1}=k_{1} / \eta=\mathrm{e}^{-t_{1} / \alpha^{\prime}}, \quad q_{2}=k_{2} / \eta=\mathrm{e}^{-t_{2} / \alpha^{\prime}}, \quad \text { and } \quad q_{3}=\eta=\mathrm{e}^{-t_{3} / \alpha^{\prime}},
$$

where the $t_{i}$ are directly related to the Schwinger proper times for the propagators depicted in Fig. 17. In this variable, the degeneration limit becomes $q_{1}<q_{2}<q_{3} \ll 1$. By using Eqs. (4.5) and (4.3) into Eqs. (4.2) and (3.24) and also (4.10), we get a very symmetric expression

$$
\begin{aligned}
\operatorname{det}\left(T_{\vec{\epsilon}^{a}}\right) & \simeq-\frac{1}{4 \pi^{2}}\left\{\left[\Gamma\left(-\epsilon_{1}^{a}\right) \Gamma\left(-\epsilon_{2}^{a}\right) \Gamma\left(\epsilon_{1}^{a}+\epsilon_{2}^{a}\right) q_{1}^{\epsilon_{1}^{a} / 2} q_{2}^{\epsilon_{2}^{a} / 2} q_{3}^{-\epsilon_{1}^{a} / 2-\epsilon_{2}^{a} / 2}\right.\right. \\
& \left.\left.\times\left(\epsilon_{1}^{a} q_{1}^{-\epsilon_{1}^{a}}+\epsilon_{2}^{a} q_{2}^{-\epsilon_{2}^{a}}-\left(\epsilon_{1}^{a}+\epsilon_{2}^{a}\right) q_{3}^{\epsilon_{1}^{a}+\epsilon_{2}^{a}}\right)\right]+\left[\epsilon_{i}^{a} \leftrightarrow-\epsilon_{i}^{a}\right]\right\},
\end{aligned}
$$


where the last square parenthesis means that we have to sum a contribution where $\epsilon_{1}, \epsilon_{2}$ have everywhere the opposite sign. From now on we choose both $\epsilon$ 's to be positive, then the dominant term in the above expression is

$$
\operatorname{det}\left(T_{\vec{\epsilon}^{a}}\right) \simeq \frac{1}{4 \pi^{2}}\left[\Gamma\left(\epsilon_{1}^{a}\right) \Gamma\left(\epsilon_{2}^{a}\right) \Gamma\left(1-\epsilon_{1}^{a}-\epsilon_{2}^{a}\right) q_{1}^{-\epsilon_{1}^{a} / 2} q_{2}^{-\epsilon_{2}^{a} / 2} q_{3}^{-\epsilon_{1}^{a} / 2-\epsilon_{2}^{a} / 2}\right]+\ldots,
$$

where the terms neglected do not contribute to the correlator among three twist fields $\sigma_{\epsilon}$ but are relevant for the couplings with excited twist fields. Let us know pose $\epsilon_{3}=1-\epsilon_{1}-\epsilon_{2}$ so that the three parameters $\epsilon_{i}$ satisfy the relation that if often considered in the twist field couplings $\sum_{i=1}^{3} \epsilon_{i}=1$. By using (4.8) and (4.2) into the integrand of Eq. (3.23) we have

$$
\begin{aligned}
\left.Z_{2}(F)\right|_{\text {uncomp. }} & \simeq \prod_{i=0}^{2}\left[\operatorname{Det}\left(1-G^{-1} \mathcal{F}_{i}\right)\left(\prod_{a=1}^{d} \sin \pi \epsilon_{i+1}^{a}\right)\right]^{1 / 2} \int \prod_{i=1}^{3}\left[q_{i}^{\epsilon_{i}-\epsilon_{i}^{2}} \frac{\Gamma\left(1-\epsilon_{i}\right)}{\Gamma\left(\epsilon_{i}\right)}\right]^{1 / 2} \\
& \times\left(\frac{1}{\ln q_{1} \ln q_{2}+\ln q_{1} \ln q_{3}+\ln q_{2} \ln q_{3}}\right)^{13-d} \prod_{i=1}^{3} \frac{d q_{i}}{q_{i}^{2}}
\end{aligned}
$$

where we used the approximate expression for $[d Z]_{g=2}[32]$ and the identity

$$
\Gamma(z) \Gamma(1-z)=\frac{\pi}{\sin \pi z} .
$$

Now we are in the position of performing the last step and factorize the degenerate surface in Fig. 1b into its constituents, as depicted in Fig. 17. In this computation we will ignore all the terms that do not depend on the world-sheet moduli. In the next section we will see that these normalizations combine nicely with others terms originating from the zero-mode contributions. The factors of $q_{i}^{\epsilon_{i} / 2-\epsilon_{i}^{2} / 2}$ in (4.9) and the measure $d q_{i} / q_{i}^{2}$ present in the untwisted $[d Z]_{g=2}$ take a simple form in the variables (4.6) and yield the measure $d t_{i} \exp \left[-M_{\epsilon_{i}}^{2} t_{i}\right]$, where $M_{\epsilon_{i}}$ is exactly the mass of the twisted tachyon (2.8) for each $\epsilon_{i}$. This term together with the leading order of the period matrix determinant have a very simple space-time interpretation: they are just a representation of the three massive propagators, with the momentum conservation present in the vertices enforced

$$
\int\left(\prod_{i=1}^{3} d t_{i}\right) \frac{\sum_{i} \exp \left[-M_{\epsilon_{i}}^{2} t_{i}\right]}{\left(t_{1} t_{2}+t_{1} t_{3}+t_{2} t_{3}\right)^{13-d}}=\int\left(\prod_{i=1}^{3} d^{26-2 d} p_{i} \frac{1}{p_{i}^{2}+M_{\epsilon_{i}}^{2}}\right) \delta\left(\sum_{i} p_{i}\right) .
$$

Thus if we compare Eq. (4.9) and Fig. 11. we can obtain an explicit expression for the square of the string vertex containing three twists fields. In formulae (and, for the moment, neglecting the normalization) we get

$$
\left\langle\sigma_{\epsilon_{1}} \sigma_{\epsilon_{2}} \sigma_{\epsilon_{3}}\right\rangle=\prod_{i=1}^{3}\left[\frac{\Gamma\left(1-\epsilon_{i}\right)}{\Gamma\left(\epsilon_{i}\right)}\right]^{1 / 4} .
$$

This result agrees with previous results [5, 8]. 


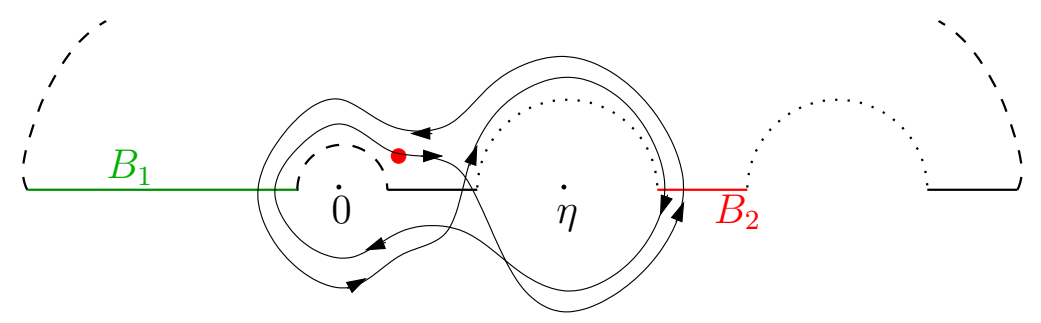

Figure 5: This represents the path of integration $\mathcal{C}$ resulting from the combination of the integrals in the square parenthesis of (4.13). Starting from the dot and following the arrows one can check that this is a closed circuit on the branched Riemann surface. The $\epsilon_{1}$-cut $\left(\epsilon_{2}\right.$-cut $)$ are along the border $B_{1}\left(B_{2}\right)$.

\subsection{Twist field couplings on a $T^{2}$}

The new ingredient in the toroidal partition function (3.29) is the exponential due to the zero-mode contribution. This exponential depends both on the twisted period matrix $T_{\bar{\epsilon}^{a}}$ we have already encountered in the previous section and on new integrals of the Prym differentials. In the two loop case (3.30) simplifies since in this case there is only one regular Prym differential and one element in the matrix $\boldsymbol{D}_{11} \equiv \boldsymbol{D}$

$$
\boldsymbol{D}=\frac{1}{2 \pi \mathrm{i}} \frac{\mathcal{S}_{1}^{-1}}{\left(1-\mathcal{S}_{2}\right)^{2}}\left[\left(1-\mathcal{S}_{2}\right) \int_{a_{1}^{-1}}-\left(1-\mathcal{S}_{1}\right) \int_{a_{2}^{-1}}\right] \frac{\left(1-\mathcal{S}_{2}^{\epsilon \cdot \tau}\right) \widehat{\boldsymbol{\Omega}}(z)}{\operatorname{det} \boldsymbol{T}} d z ;
$$

moreover in (3.29) $\alpha_{0} \equiv \alpha_{0}^{\mu=1}$ is the left moving momentum (2.13) running in the first loop. Each integral over the $a_{1}$ and the $a_{2}$ cycle does not follow a close path because of the monodromies of $\widehat{\Omega}$. However, from our analysis in the closed string channel (3.12), we know that the combination of integrals in the square parenthesis is independent of the starting point. Thus this combination should represent a closed path on the branched Riemann surface. In fact, if we follow the cycle $a_{2}^{-1}$, we have to through the cut $B_{2}$, and the integrand is multiplied by $\mathcal{S}_{2}$. So it is smoothly connected to the last integral $\mathcal{S}_{2} \int_{a_{1}^{-1}}$ and picks a phase $\mathcal{S}_{1}$ when passes through the cut $B_{1}$ following $a_{1}^{-1}$. Then the result is smoothly connected to the second integral after we transformed the anti-clockwise orientation of $a_{2}^{-1}$ into the clockwise one $\left(a_{2}\right)$. In presence of the cut along $B_{2}$ we have $\mathcal{S}_{1} \int_{a_{2}}=-\mathcal{S}_{1} \mathcal{S}_{2} \int_{a_{2}^{-1}}$. Repeating the same steps we see that the third and last integral brings back the integrand on the sheet where we started from, thus showing that the path of Fig. 15 is closed. At leading order in the limit (4.1), the integrand $\widehat{\Omega}$ is defined through the expansions in (4.2), where this time we can neglect all the terms in the square parenthesis. In fact, contrary to what happened in (4.3), the path of integration depicted in Fig. 5 never comes close to the points 0 and $\eta=q_{3}$. After we truncated the integrand 
in (4.13) at the first order we are free to deform the contour to the real axis obtaining

$$
\left[\left(1-\mathcal{S}_{1}\right) \int_{a_{2}^{-1}}-\left(1-\mathcal{S}_{2}\right) \int_{a_{1}^{-1}}\right]=\left(1-\mathcal{S}_{1}\right)\left(1-\mathcal{S}_{2}\right) \int_{0}^{q_{3}} .
$$

By using this relation in (4.13) we have

$$
\boldsymbol{D}=-\frac{1}{2 \pi \mathrm{i}} \frac{1-\mathcal{S}_{1}^{-1}}{1-\mathcal{S}_{2}} \frac{\boldsymbol{f}}{\operatorname{det} \boldsymbol{T}},
$$

where the function $\boldsymbol{f}$ is the integral between 0 and $q_{3}$ of the Prym differential $\left(1-\mathcal{S}_{2}^{\epsilon \cdot \tau}\right) \widehat{\boldsymbol{\Omega}}$ approximated to the first order. From (4.2) and using that $z \ll 1$, we have

$$
f(\epsilon) \sim q_{1}^{-\frac{\epsilon_{1}}{2}} q_{2}^{-\frac{\epsilon_{2}}{2}} q_{3}^{\frac{\epsilon_{1}+\epsilon_{2}}{2}} \int_{0}^{q_{3}}\left[\frac{q_{1}^{\epsilon_{1}} q_{3}^{\epsilon_{1}+\epsilon_{2}}-1}{z-q_{3}} q_{2}^{\epsilon_{2}}-\frac{q_{2}^{\epsilon_{2}} q_{3}^{\epsilon_{1}+\epsilon_{2}}-1}{z} q_{1}^{\epsilon_{1}}\right] \frac{\mathrm{e}^{\pi \mathrm{i}\left(\epsilon_{1}+\epsilon_{2}\right)} d z}{\left(q_{3}-z\right)^{\epsilon_{2}} z^{\epsilon_{1}}},
$$

where $f(\epsilon)$ indicates the first component of the space-time matrix $\boldsymbol{f}$ that is diagonal in the complex basis. The other component is, of course, $f(-\epsilon)$ where the signs of all twists are reversed. After the change of variable $y=z / q_{3}$, this integral reduces to the integral definition of the Euler Beta. Then, by using (4.10), we obtain a simple expression for $\boldsymbol{D}$, valid in the limit (4.1)

$$
\begin{aligned}
\boldsymbol{D} & \sim\left\{\frac{\sin \left(\pi \epsilon_{1}+\pi \epsilon_{2}\right) \sin \left(\pi \epsilon_{1}\right)}{\pi \sin \left(\pi \epsilon_{2}\right)} q_{1}^{\epsilon_{1} / 2} q_{2}^{\epsilon_{2} / 2} q_{3}^{-\epsilon_{1} / 2-\epsilon_{2} / 2} \Gamma\left(-\epsilon_{1}\right) \Gamma\left(-\epsilon_{2}\right) \Gamma\left(\epsilon_{1}+\epsilon_{2}\right)\right. \\
& \left.\times \frac{\left(\epsilon_{1} q_{1}^{-\epsilon_{1}}+\epsilon_{2} q_{2}^{-\epsilon_{2}}-\left(\epsilon_{1}+\epsilon_{2}\right) q_{3}^{\epsilon_{1}+\epsilon_{2}}\right)}{-2 \pi \operatorname{idet} T_{\vec{\epsilon}}}, \epsilon_{i} \leftrightarrow-\epsilon_{i}\right\} .
\end{aligned}
$$

By using (4.7), one can check that

$$
D(\epsilon)-D(-\epsilon)=-2 \mathrm{i} \frac{\sin \left(\pi \epsilon_{1}\right) \sin \left(\pi \epsilon_{1}+\pi \epsilon_{2}\right)}{\sin \left(\pi \epsilon_{2}\right)},
$$

which is consistent with the general property $(3.21) 9$.

The first order of the $q_{i} \rightarrow 0$ limit describes the factorization depicted in Fig. 11. We can now use the result we have and check that the leading term in the exponential (4.13) does not depend on the $q_{i}$ 's. By using also (3.18) and (3.19) we obtain

Eq. (4.13) $\sim \exp \left\{-\frac{\pi}{2}\left(m^{1}+\bar{U} m^{2}\right)\left[\frac{\sin \left(\pi \epsilon_{1}\right) \sin \left(\pi \epsilon_{1}+\pi \epsilon_{2}\right)}{\sin \left(\pi \epsilon_{2}\right)} \frac{\left|\mathrm{i}\left(f_{1}-T_{1}\right)+T_{2}\right|^{2}}{T_{2} U_{2}}\right]\left(m^{1}+U m^{2}\right)\right\}$.

\footnotetext{
${ }^{9}$ Remember that here $\epsilon_{2}$ is the opposite of the one used in the previous Section, as discussed at the beginning of Sect. 4.1.
} 
In the $T^{2}$ case under analysis, it is easy to relate the eigenvalues of $\mathcal{S}_{\mu}$ and the phases $\epsilon_{\mu}$

$$
\mathrm{e}^{2 \pi \mathrm{i} \epsilon_{2}}=\frac{T-f_{0}}{\bar{T}-f_{0}} \frac{\bar{T}-f_{2}}{T-f_{2}} \quad, \quad \mathrm{e}^{2 \pi \mathrm{i} \epsilon_{1}}=\frac{T-f_{1}}{\bar{T}-f_{1}} \frac{\bar{T}-f_{0}}{T-f_{0}} .
$$

From this one can check that in the square parenthesis in (4.19) all terms containing the Kähler moduli $T_{1}$ and $T_{2}$ disappear and one has

$$
\left[\frac{\sin \left(\pi \epsilon_{1}\right) \sin \left(\pi \epsilon_{1}+\pi \epsilon_{2}\right)}{\sin \left(\pi \epsilon_{2}\right)} \frac{\left|\mathrm{i}\left(f_{1}-T_{1}\right)+T_{2}\right|^{2}}{T_{2}}\right]=\frac{\left|f_{1}-f_{0}\right|\left|f_{1}-f_{2}\right|}{\left|f_{2}-f_{0}\right|} .
$$

Thus at the leading order, the open string channel partition function (3.29) becomes

$$
\begin{aligned}
Z_{2}(F) & \simeq \sqrt{G} \mathrm{e}^{\phi_{10}} \prod_{i=0}^{2}\left[\operatorname{Det}\left(1-G^{-1} \mathcal{F}_{i}\right)\left(\prod_{a=1}^{d} \sin \pi \epsilon_{i+1}^{a}\right)\right]^{1 / 2} \sum_{m} \delta\left[\left(1-\mathcal{S}_{\mu}\right) \alpha_{0}^{\mu}\right] \\
& \times \exp \left\{-\frac{\pi}{2}\left(m^{1}+\bar{U} m^{2}\right) \frac{\left|f_{1}-f_{0}\right|\left|f_{1}-f_{2}\right|}{\left|f_{2}-f_{0}\right|} \frac{1}{U_{2}}\left(m^{1}+U m^{2}\right)\right\} \\
& \times \prod_{i=1}^{3}\left[\frac{\Gamma\left(1-\epsilon_{i}\right)}{\Gamma\left(\epsilon_{i}\right)}\right]^{1 / 2} \int\left(\prod_{i=1}^{3} d^{26-2 d} p_{i} \frac{1}{p_{i}^{2}+M_{\epsilon_{i}}^{2}}\right) \delta\left(\sum_{i} p_{i}\right)
\end{aligned}
$$

where we wrote explicitly also the usual $F_{i}$-independent normalizations contained in $[d Z]_{2}$ : the volume $(\sqrt{G})$ and the dilaton factors $\left(\mathrm{e}^{\phi_{10}}\right)$. It is useful to rewrite the latter in terms of the effective $d$-dimensional dilaton according to $\mathrm{e}^{2 \phi_{10}}=\sqrt{G} \mathrm{e}^{2 \phi_{d}}$. Then the product over $\sin \pi \epsilon_{i}$ can be written as follows

$$
\prod_{i=1}^{3} \prod_{a=1}^{d} \sin ^{2} \pi \epsilon_{i}^{a}=\prod_{i=1}^{3} \operatorname{Det}\left(\frac{1-\mathcal{S}_{i}}{2}\right)=\prod_{i=0}^{2} \operatorname{Det}\left(\frac{R_{i+1}-R_{i}}{2}\right),
$$

where in the last step we used the fact the the reflection matrices $R_{i}$ are even dimensional and have unit determinant (we also take a cyclic convention $R_{3} \equiv R_{0}$ ). By using (3.1) and (4.23) into (4.9), we see that this determinant combines nicely with the Born-Infeld normalization yielding

$$
\prod_{i=0}^{2}\left[\operatorname{Det}\left(1-G^{-1} \mathcal{F}_{i}\right) \prod_{a=1}^{d} \sin \pi \epsilon_{i+1}^{a}\right]^{1 / 2}=(\operatorname{Det} G)^{-3 / 4} \prod_{i=0}^{2}\left[\operatorname{Det}\left(F_{i+1}-F_{i}\right)\right]^{1 / 4}
$$

Let us now analyze the second line of (4.22) and, for sake of simplicity, suppose that all $\left(f_{i}-f_{j}\right)$ 's which are integers as consequence of (2.1)-(3.1), are coprime. Then the Kronecker delta implies that

$$
m_{1}^{M} I_{10}+m_{2}^{M} I_{20}=0 \Rightarrow m_{1}^{M} \equiv m^{M}=I_{20} h^{M}
$$


where $I_{\mu 0}=f_{\mu}-f_{0}=p_{\mu}-p_{0}, h^{M} \in Z$ and $M=1,2$. So the exponential terms form a 2-dimensional Theta function whose modular parameter contains the complex structure and the product $\mathcal{I}=\left|I_{10} I_{02} I_{21}\right|$; notice that $\mathcal{I}=2 \mathcal{I}^{\prime}$ is even because it is the absolute value of the product of three integers summing to zero. At this point, we rewrite the sum on $h^{1}$ by performing a Poisson resummation

$$
\sum_{h^{1}=-\infty}^{\infty} \mathrm{e}^{-\pi A\left(h^{1}\right)^{2}+2 \pi h^{1} A s}=\frac{1}{\sqrt{A}} \mathrm{e}^{\pi A s^{2}} \sum_{h^{1}=-\infty}^{\infty} \mathrm{e}^{-\pi\left(h^{1}\right)^{2} / A-2 \pi \mathrm{i} h^{1} s} .
$$

The second line of (4.22) becomes

$$
\sqrt{\frac{U_{2}}{\mathcal{I}^{\prime}}} \sum_{h^{1}, h^{2}} \exp \left[-\frac{\pi\left(h^{1}\right)^{2} U_{2}}{\mathcal{I}^{\prime}}+2 \pi \mathrm{i} h^{1} h^{2} U_{1}-\pi \mathcal{I}^{\prime} U_{2}\left(h^{2}\right)^{2}\right] .
$$

In this form we can decouple the two sums. However, it is first convenient to break the sum over $h^{1}$ in $\mathcal{I}^{\prime}$ sums over integers of the form $\mathcal{I}^{\prime} \tilde{h}^{1}+\ell^{\prime}$, with $\ell^{\prime}=0,1, \ldots, \mathcal{I}^{\prime}-1$. Then we can introduce an other set of integers $r, s$ : $\tilde{h}^{1}-h^{2}=r$ and $\tilde{h}^{1}+h^{2}=s$, which are however constrained to have the same parity. Therefore the sum over $r, s$ breaks into two parts, one over $r=2 k, s=2 l$ and the other over $r=2 k+1, s=2 l+1$. This second sum can be obtained from the first one simply by replacing $\ell^{\prime}$ with $\mathcal{I}^{\prime}+\ell^{\prime}$. Then we can finally rewrite (4.27) in terms of 1-dimensional Theta function 10 .

$$
\sqrt{\frac{2 U_{2}}{\mathcal{I}}} \sum_{\ell=0}^{\mathcal{I}-1} \sum_{l, k} \exp \left[\pi \mathrm{i} \mathcal{I}\left(l+\frac{\ell}{\mathcal{I}}\right)^{2} U\right] \exp \left[-\pi \mathrm{i} \mathcal{I}\left(k+\frac{\ell}{\mathcal{I}}\right)^{2} \bar{U}\right] .
$$

Thus we see that in the limit (4.1) the full twisted partition factorizes into a sum of diagrams like the one depicted in Fig. 1k, and each diagram is labeled by a different $\ell$. However this sum was expected and is due to the finite dimensional degeneracy of each twisted string state, as was quickly mentioned in Section 2 in relations to the zero-modes. From a lower dimensional point of view these states are seen as different particles of the same family, since they have the same quantum numbers. Since for each twisted string this degeneracy is exactly $\left|I_{i j}\right|$, the number of possible three point functions is $\mathcal{I}$ in agreement with our interpretation of (4.28). Thus we can focus on each value of $\ell$ separately and derive from (4.22) the expression for the 3-twist field correlator on a $T^{2}$

$$
\left\langle\sigma_{\epsilon_{1}} \sigma_{\epsilon_{2}} \sigma_{\epsilon_{3}}\right\rangle_{\ell}=\left[\prod_{i=1}^{3} \frac{\Gamma\left(1-\epsilon_{i}\right)}{\Gamma\left(\epsilon_{i}\right)}\right]^{1 / 4} \mathrm{e}^{\phi_{d} / 2}\left(2 U_{2}\right)^{1 / 4} \sum_{l=-\infty}^{\infty} \exp \left\{\pi \mathrm{i} \mathcal{I}\left(l+\frac{\ell}{\mathcal{I}}\right)^{2} U\right\}
$$

where the overall normalization assumes a simple form since $F$-dependent part of (4.24) exactly cancels the factor of $\sqrt{\mathcal{I}}$ in (4.28). For small $\epsilon$ we reproduce the result of [22],

\footnotetext{
${ }^{10}$ Notice that now the summation index $\ell$ runs from 0 to $\mathcal{I}-1$.
} 
including the U-dependent normalization. If we exchange $T$ and $U$ in (4.29), we pass to the T-dual setup of D-branes at angle and find agreement with the results of [7, 8]. In [7, the amplitudes corresponding to different families were labeled by three integers $i, j, k$, but it can be checked that their set of Yukawa couplings corresponds exactly to our (4.29).

\section{Acknowledgments}

This work is partially supported by the European Community's Human Potential under contract MRTN-CT-2004-005104 and MRTN-CT-2004-512194, and by the Italian MIUR under contract PRIN 2005023102. We wish to thank Dario Duò and Lorenzo Magnea for collaboration on related topics and for helpful discussions.

\section{A The twisted sewing on $T^{2 d}$}

In the operator formalism all the basic building blocks like string vertices and propagators are written in terms of the Schottky group. In both the closed and the open string description this group is generated by $2 \times 2$ matrices that act on the complex plane as follows

$$
\gamma(z)=\left(\begin{array}{ll}
a & b \\
c & d
\end{array}\right)\left(\begin{array}{l}
z \\
1
\end{array}\right)=\frac{a z+b}{c z+d}, \quad \text { with } \quad a d-b c=1 .
$$

In the string vertices the bosonic oscillators $a_{n}$ or $\tilde{a}_{n}$ are naturally contracted with the following infinite dimensional representation of the Schottky group (here $n, m \neq 0$ )

$$
\begin{array}{rlrl}
D_{n m}(\gamma)=\left.\frac{1}{m !} \sqrt{\frac{m}{n}} \partial_{z}^{m} \gamma^{n}\right|_{z=0} & , & D_{00} & =-\ln d \\
D_{n 0}=\frac{1}{\sqrt{n}} \gamma^{n}(0) & , & D_{0 m}=\left.\frac{\sqrt{m}}{2 m !} \partial_{z}^{m} \ln \gamma^{\prime}\right|_{z=0}=\frac{1}{\sqrt{m}}\left(\frac{1}{\gamma^{-1}(\infty)}\right)^{m} .
\end{array}
$$

As already noticed in the main text, this is not a true representation when the zero modes are included. In fact the product law is

$$
D_{n m}\left(\gamma_{1} \gamma_{2}\right)=\sum_{l=1}^{\infty} D_{n l}\left(\gamma_{1}\right) D_{l m}\left(\gamma_{2}\right)+D_{n 0}\left(\gamma_{1}\right) \delta_{m 0}+D_{0 m}\left(\gamma_{2}\right) \delta_{n 0}
$$

This is the source of some complication in the zero-mode computation of the twisted planar partition function and in this Appendix we want to give some details on the technical steps leading to (3.7). 
The closed string vertex describing the emission of $g+1$ off-shell strings can be written in terms of $g+1$ transformations (A.1) $V_{i}$

$$
\begin{aligned}
\mathbf{V}_{g+1} & \sim \prod_{i=0}^{g}\left[\sum_{n^{i}, w^{i}}\left\langle n^{i}, w^{i} ; 0\right|\right] \exp \left[-\sum_{i<j=0}^{g} \sum_{k, l=0}^{\infty} a_{k}^{i} D_{k l}\left(\Gamma V_{i}^{-1} V_{j}\right) G a_{l}^{j}\right] \\
& \times \exp \left[-\sum_{i<j=0}^{g} \sum_{k, l=0}^{\infty} \tilde{a}_{k}^{i} D_{k l}\left(\Gamma \bar{V}_{i}^{-1} \bar{V}_{j}\right) G \tilde{a}_{l}^{j}\right] \delta\left(\sum_{i=0}^{g} a_{0}^{i}\right) \delta\left(\sum_{j=0}^{g} \tilde{a}_{0}^{j}\right),
\end{aligned}
$$

where the $\delta$ 's should be interpreted as Kronecker delta's and we neglected the overall normalization. Here the $V_{i}(z)$ 's are local coordinates around each puncture which is placed at $V_{i}(0)=z_{i}, \Gamma$ is the inversion $\Gamma(z)=1 / z$, and since we are working in the parameterization of Fig 2 the right-moving coordinates are just the complex conjugate of the left moving ones. Of course when (A.4) is saturated with $g+1$ on-shell states $V_{g+1}$ in order to compute a tree-level amplitude the dependence on the local coordinates disappears. However our aim is to saturate all legs of (A.4) with boundary states; in this case the $V_{i}$ 's play a role, because they yield the generators of the Schottky group (3.2) that defines the surface of Fig. 2. Also the boundary states are written in terms of the matrices $D_{n m}$

$$
\begin{aligned}
& \left|B_{i}\right\rangle \sim \sqrt{\operatorname{Det}\left(1-G^{-1} \mathcal{F}_{i}\right)} \int_{0}^{1} \frac{d x}{x(1-x)} \delta^{d}\left(\tilde{a}_{0}+R_{i} a_{0}\right)\left|B_{i}(x)\right\rangle, \\
& \left|B_{i}(x)\right\rangle=\exp \left[-\sum_{n, m=0}^{\infty} \tilde{a}_{n}^{\dagger} D_{n m}(P(x)) G R_{i} a_{m}^{\dagger}\right] \mathrm{e}^{2 \pi \mathrm{i} \sqrt{\alpha^{\prime}}\left(A_{i}\right)_{M} w_{i}^{M}}\left(\sum_{n, w}|n, w ; 0\rangle\right),
\end{aligned}
$$

where again we neglected all $F_{i}$ independent normalizations, which can be found in [17] or restored at the end by requiring that the partition function is proportional to the total volume and the appropriate power of the string coupling. The phase depending on the Wilson line $A_{i}$ is just the rewriting in terms of the winding modes of the usual term $\exp \left\{\mathrm{i} A_{M} \oint d x^{M}\right\}$ appearing in the open string action for constant gauge fields. With a small abuse of notation we indicate with $\left|B_{i}\right\rangle$ both the standard boundary state (2.12) whose effect is just to identify left and right moving modes and the boundary state (A.5) that contains also a string propagator. This propagator is contained in the transformation $P(x)$, which is also of the type (A.1) and depends on a single real variable $x$. As we will see the specific form chosen for $P(x)$ and the $V_{i}$ 's is not relevant for our purposes.

The insertion of the first boundary states $\left|B_{0}\right\rangle$ has the effect of mixing left and the right moving modes. This can be easily seen for the non-zero mode sector, where the boundary state simply transforms the $\tilde{a}_{m}^{0}$ into creation operators $a_{m}^{0 \dagger}$. When the scalar product over the $i=0$ Hilbert space is computed, the exponential factors at the left containing the 
destruction modes $a_{m}^{0}$ are glued together with the exponentials at the right containing the creation modes $a_{m}^{0 \dagger}$. The zero-mode sector has to be treated separately and by using the momentum and winding conservations present in (A.4) we can re-express $a_{0}^{0}$ in terms of the $a_{0}^{\mu}$, with $\mu=1, \ldots, g$. Then by using the product law A.3 and the properties summarized in Appendix B of [15] we get a vertex describing the emission of $g$ closed strings from a disk represented by the boundary state $\left|B_{0}\right\rangle$

$$
\begin{aligned}
\mathbf{V}_{g ; 1} & \sim \prod_{i=1}^{g}\left[\sum_{n^{i}, w^{i}}\left\langle n^{i}, w^{i} ; 0\right|\right] \delta\left[\sum_{\mu=1}^{g}\left(a_{0}^{\mu}+R_{0}^{-1} \tilde{a}_{0}^{\mu}\right)\right] \exp \left[-\sum_{\mu, \nu=1}^{g} a_{k}^{\mu} D_{k l}\left(U_{\mu} \bar{V}_{\nu}\right) G R_{0}^{-1} \tilde{a}_{l}^{\nu}\right] \\
& \times \exp \left[-\sum_{\mu<\nu=1}^{g} a_{k}^{\mu} D_{k l}\left(U_{\mu} V_{\nu}\right) G a_{l}^{\nu}\right] \exp \left[-\sum_{\mu<\nu=1}^{g} \tilde{a}_{k}^{\mu} D_{k l}\left(\bar{U}_{\mu} \bar{V}_{\nu}\right) G \tilde{a}_{l}^{\nu}\right]
\end{aligned}
$$

where we have understood the sums over the indices $k, l$ from 0 to $\infty$ and followed the notation of [15], where $U=\Gamma V^{-1}$. We also used the freedom to redefine the local coordinates $V_{\mu}$ by a similarity transformation to eliminate any dependence on $V_{0}$.

At this point we can insert the remaining $g$ boundary states in (A.6) and use again the properties of Canonical Forms [15] and Eq. (A.3. to rewrite the exponentials in a normal ordered way

$$
\begin{aligned}
\mathbf{V}_{g+1 ; 0} & \sim \prod_{i=1}^{g}\left[\sum_{n^{i}, w^{i}}\left\langle n^{i}, w^{i} ; 0\right|\right] \delta\left[\sum_{\mu=1}^{g}\left(1-\mathcal{S}_{\mu}\right) a_{0}^{\mu}\right] \exp \left[-\frac{1}{2} \sum_{\mu \neq \nu=1}^{g} a_{k}^{\mu} D_{k l}\left(U_{\mu} V_{\nu}\right) G a_{l}^{\nu}\right] \\
& \times \quad \exp \left[\sum_{\mu, \nu=1}^{g} a_{k}^{\mu} D_{k l}\left(U_{\mu} \tilde{\bar{V}}_{\nu}\right) G \mathcal{S}_{\nu} a_{l}^{\nu \dagger}\right]: \\
& \times \exp \left[-\frac{1}{2} \sum_{\mu \neq \nu=1}^{g} a_{k}^{\mu \dagger} D_{k l}\left(\tilde{\bar{U}}_{\mu} \tilde{\bar{V}}_{\nu}\right) G \mathcal{S}_{\mu}^{-1} \mathcal{S}_{\nu} a_{l}^{\nu \dagger}\right] \prod_{i=1}^{g}\left[\sum_{n^{i}, w^{i}}\left|n^{i}, w^{i} ; 0\right\rangle\right],
\end{aligned}
$$

where the tilded local coordinates are related to the original ones by $\tilde{V}=V P$. Then we need to compute the scalar products over the oscillator modes of all Hilbert spaces. This can be done more easily by inserting the identity operator written in terms of coherent states between the creation and the destruction modes [15]. This has the effect of transforming the scalar product over the non-zero modes into a Gaussian integral which yields the following contribution to $\mathbf{V}_{g+1}$

$$
\{\operatorname{Det}[G(1-H)]\}^{-1 / 2} \exp \left[\frac{1}{2}\left(B_{2}, B_{1}\right)(1-H)^{-1} G^{-1}\left(\begin{array}{c}
C_{2} \\
C_{1}
\end{array}\right)\right]
$$

where the determinant is over all indices (space-time, loop and oscillator indices). The 
definitions of $B, C$ and $H$ are:

$$
\begin{gathered}
H=\left(\begin{array}{cc}
D_{n m}\left(U_{\mu} \tilde{\bar{V}}_{\nu}\right) \mathcal{S}_{\nu} & -D_{n m}\left(U_{\mu} V_{\nu}\right) \\
-D_{n m}\left(\tilde{\bar{U}}_{\mu} \tilde{\bar{V}}_{\nu}\right) \mathcal{S}_{\mu}^{-1} \mathcal{S}_{\nu} & D_{n m}\left(\tilde{\bar{U}}_{\mu} V_{\nu}\right) \mathcal{S}_{\mu}^{-1}
\end{array}\right) \\
\left(B_{1}\right)_{m}^{\nu}=\sum_{\substack{1 \leq \mu \leq g \\
1 \leq k<\infty}} a_{0}^{\mu} G\left[-D_{0 k}\left(U_{\mu}\right)+D_{0 k}\left(\tilde{\bar{U}}_{\mu}\right) \mathcal{S}_{\mu}^{-1}+\delta_{\mu \nu} D_{0 k}\left(U_{\mu}\right)\right] D_{k m}\left(V_{\nu}\right)+a_{0}^{\nu} G D_{0 m}\left(V_{\nu}\right) \\
\left(B_{2}\right)_{m}^{\nu}=\sum_{\substack{1 \leq \mu \leq g \\
1 \leq k<\infty}} a_{0}^{\mu} G\left[D_{0 k}\left(U_{\mu}\right)-D_{0 k}\left(\tilde{\bar{U}}_{\mu}\right) \mathcal{S}_{\mu}^{-1}+\delta_{\mu \nu} D_{0 k}\left(\tilde{\bar{U}}_{\mu}\right) \mathcal{S}_{\mu}^{-1}\right] D_{k m}\left(\tilde{\bar{V}}_{\nu}\right) \mathcal{S}_{\nu}+a_{0}^{\nu} G D_{0 m}\left(\tilde{\bar{V}}_{\nu}\right) \\
\left(C_{2}\right)_{m}^{\nu}=\sum_{\substack{1 \leq \mu \leq g \\
1 \leq k<\infty}} D_{m k}\left(U_{\nu}\right) G\left[-D_{k 0}\left(V_{\mu}\right)+\mathcal{S}_{\mu} D_{k 0}\left(\tilde{\bar{V}}_{\mu}\right)+\delta_{\mu \nu} D_{k 0}\left(V_{\mu}\right)\right] a_{0}^{\nu}+D_{m 0}\left(U_{\nu}\right) G a_{0}^{\nu} \\
\left(C_{1}\right)_{m}^{\nu}=\sum_{\substack{1 \leq \mu \leq g \\
1 \leq k<\infty}} D_{m k}\left(\tilde{\bar{U}}_{\nu}\right) G \mathcal{S}_{\nu}^{-1}\left[D_{k 0}\left(V_{\mu}\right)-D_{k 0}\left(\tilde{\bar{V}}_{\mu}\right) \mathcal{S}_{\mu}+\delta_{\mu \nu} D_{k 0}\left(\tilde{\bar{U}}_{\mu}\right) \mathcal{S}_{\mu}\right] a_{0}^{\mu}+D_{m 0}\left(\tilde{\bar{U}}_{\nu}\right) G a_{0}^{\nu} .
\end{gathered}
$$

Notice that in the expressions for $B_{2}$ and $C_{1}$, that follow from (A.7) and (A.3), using also (3.6) , the string propagators $P$ contained in the tilded local coordinates are not always paired with the corresponding space-time matrix $\mathcal{S}$. This is the origin of the subtlety mentioned in Section (3.1). At this point one needs to combine the result (A.8) with the zero-mode part of Eq. (A.6) and keep following the steps of Appendix D of [15], now also with the space-time matrices $\mathcal{S}$. In the present case the combination of local coordinate representing the generators of the Schottky group is $S_{\mu}^{\mathrm{cl}} \equiv \tilde{\bar{V}}_{\mu} U_{\mu}$. By using many times the group property (A.3) we arrive to formulae analogous to (D.21) and then (D.24) of [15]. In particular, we find that the matrix $C_{\mu \nu}^{(1)}$ in our (3.7) is

$$
\begin{aligned}
C_{\mu \nu}^{(1)} & =\left(D_{00}\left(S_{\mu}\right)+D_{00}\left(S_{\mu}^{-1}\right)\right) \delta_{\mu \nu} \\
& +{ }^{(-\mu)} \sum_{\alpha}{ }^{(-\nu)} D_{0 n}\left(S_{\mu}\right) D_{n m}\left(T_{\alpha}\right) D_{m 0}\left(S_{\nu}\right) \mathcal{T}_{\alpha} \mathcal{S}_{\nu} \\
& -{ }^{(-\mu)} \sum_{\alpha}{ }^{\prime(+\nu)} D_{0 n}\left(S_{\mu}\right) D_{n m}\left(T_{\alpha}\right) D_{m 0}\left(S_{\nu}^{-1}\right) \mathcal{T}_{\alpha} \\
& -{ }^{(+\mu)} \sum_{\alpha}{ }^{\prime}{ }^{(-\nu)} D_{0 n}\left(S_{\mu}^{-1}\right) D_{n m}\left(T_{\alpha}\right) D_{m 0}\left(S_{\nu}\right) \mathcal{S}_{\mu}^{-1} \mathcal{T}_{\alpha} \mathcal{S}_{\nu} \\
& +{ }^{(+\mu)} \sum_{\alpha}^{(+\nu)} D_{0 n}\left(S_{\mu}^{-1}\right) D_{n m}\left(T_{\alpha}\right) D_{m 0}\left(S_{\nu}^{-1}\right) \mathcal{S}_{\mu}^{-1} \mathcal{T}_{\alpha},
\end{aligned}
$$

where now the indices $n, m$ run from 1 to $\infty$ and the superscript "cl" on the elements $S_{\mu}$ and $T_{\alpha}$ of the Schottky groups is understood. The sums with the superscripts $( \pm \mu)$ at the left and $( \pm \nu)$ at the right are taken over all elements $T_{\alpha}$ of the Schottky group which contain neither $S_{\mu}^{ \pm n}$ as leftmost factor nor $S_{\nu}^{ \pm n}$ as rightmost factor for any positive integer $n$; moreover the prime on the sum means that the identity is absent when $\mu=\nu$. Finally 
the matrix $\mathcal{T}_{\alpha}$ is the space-time analogue of the Schottky element $T_{\alpha}$ and is the product of the $\mathcal{S}_{\mu}$ corresponding to the generators contained in $T_{\alpha}$. Then by using the explicit expressions for the D's (A.2), one can check that (A.10) is just the series expansion of the following logarithms

$$
\begin{aligned}
C_{\mu \nu}^{(1)} & =-\delta_{\mu \nu} \ln \left(a_{\mu} d_{\mu}\right)+{ }^{(-\mu)} \sum_{\alpha}^{(-\nu)} \ln \left[\frac{S_{\mu}^{-1}(\infty)-T_{\alpha}(0)}{S_{\mu}^{-1}(\infty)-T_{\alpha} S_{\nu}(0)}\right] \mathcal{T}_{\alpha} \mathcal{S}_{\nu} \\
& -{ }^{(-\mu)} \sum_{\alpha}{ }^{(+\nu)} \ln \left[\frac{S_{\mu}^{-1}(\infty)-T_{\alpha}(0)}{S_{\mu}^{-1}(\infty)-T_{\alpha} S_{\nu}^{-1}(0)}\right] \mathcal{T}_{\alpha} \\
& -{ }^{(+\mu)} \sum_{\alpha}{ }^{\prime(-\nu)} \ln \left[\frac{S_{\mu}(\infty)-T_{\alpha}(0)}{S_{\mu}(\infty)-T_{\alpha} S_{\nu}(0)}\right] \mathcal{S}_{\mu}^{-1} \mathcal{T}_{\alpha} \mathcal{S}_{\nu} \\
& +{ }^{(+\mu)} \sum_{\alpha}^{(+\nu)} \ln \left[\frac{S_{\mu}(\infty)-T_{\alpha}(0)}{S_{\mu}(\infty)-T_{\alpha} S_{\nu}^{-1}(0)}\right] \mathcal{S}_{\mu}^{-1} \mathcal{T}_{\alpha} .
\end{aligned}
$$

Finally one can check that this expression agrees with the one of (3.7) given in terms of integrals of the differentials $\boldsymbol{\zeta}_{\nu}$ 's when the base point is placed at infinity $w=\infty$. However, as proved in the main text, that combination of integrals is independent of this particular choice, so finally we can write $C_{\mu \nu}^{(1)}$ as in Eq. (3.7).

\section{References}

[1] L. J. Dixon, J. A. Harvey, C. Vafa, and E. Witten, Nucl. Phys. B261, 678 (1985).

L. J. Dixon, J. A. Harvey, C. Vafa, and E. Witten, Nucl. Phys. B274, 285 (1986).

[2] M. Berkooz, M. R. Douglas, and R. G. Leigh, Nucl. Phys. B480, 265 (1996), hep-th/9606139.

[3] A. Abouelsaood, J. Callan, Curtis G., C. R. Nappi, and S. A. Yost, Nucl. Phys. B280, 599 (1987).

[4] L. J. Dixon, D. Friedan, E. J. Martinec, and S. H. Shenker, Nucl. Phys. B282, 13 (1987).

[5] T. T. Burwick, R. K. Kaiser, and H. F. Muller, Nucl. Phys. B355, 689 (1991).

J. Erler, D. Jungnickel, M. Spalinski, and S. Stieberger, Nucl. Phys. B397, 379 (1993), hep-th/9207049.

S. Stieberger, D. Jungnickel, J. Lauer, and M. Spalinski, Mod. Phys. Lett. A7, 3059 (1992), hep-th/9204037.

S. Stieberger, Phys. Lett. B300, 347 (1993), hep-th/9211027. 
[6] E. Gava, K. S. Narain, and M. H. Sarmadi, Nucl. Phys. B504, 214 (1997), hep-th/9704006.

[7] D. Cremades, L. E. Ibanez, and F. Marchesano, JHEP 07, 038 (2003), hep-th/0302105.

[8] M. Cvetic and I. Papadimitriou, Phys. Rev. D68, 046001 (2003), hep-th/0303083.

S. A. Abel and A. W. Owen, Nucl. Phys. B663, 197 (2003), hep-th/0303124.

S. A. Abel and A. W. Owen, Nucl. Phys. B682, 183 (2004), hep-th/0310257.

D. Lust, P. Mayr, R. Richter, and S. Stieberger, Nucl. Phys. B696, 205 (2004), hep-th/0404134.

[9] S. Kachru and E. Silverstein, JHEP 01, 004 (1999), hep-th/9810129.

R. Iengo and C.-J. Zhu, JHEP 04, 028 (2000), hep-th/9912074.

K. Aoki, E. D'Hoker, and D. H. Phong, Nucl. Phys. B688, 3 (2004), hep-th/0312181.

[10] C. Bachas and M. Porrati, Phys. Lett. B296, 77 (1992), hep-th/9209032.

[11] C. Bachas, Phys. Lett. B374, 37 (1996), hep-th/9511043.

M. Billo, P. Di Vecchia, and D. Cangemi, Phys. Lett. B400, 63 (1997), hep-th/9701190.

[12] I. Antoniadis, K. S. Narain, and T. R. Taylor, Nucl. Phys. B729, 235 (2005), hep-th/0507244.

[13] A. Dabholkar and D. Gaiotto, (2006), hep-th/0612011.

[14] L. Alvarez-Gaume, C. Gomez, G. W. Moore, and C. Vafa, Nucl. Phys. B303, 455 (1988).

[15] P. Di Vecchia, F. Pezzella, M. Frau, K. Hornfeck, A. Lerda and A. Sciuto, Nucl. Phys. B322, 317 (1989).

[16] P. Di Vecchia and A. Liccardo, (1999), hep-th/9912275.

[17] M. Frau, I. Pesando, S. Sciuto, A. Lerda, and R. Russo, Phys. Lett. B400, 52 (1997), hep-th/9702037.

[18] R. Russo and S. Sciuto, Nucl. Phys. B669, 207 (2003), hep-th/0306129.

[19] R. Russo and S. Sciuto, Fortsch. Phys. 52, 678 (2004), hep-th/0312205. 
[20] J. J. Atick, L. J. Dixon, P. A. Griffin, and D. Nemeschansky, Nucl. Phys. B298, 1 (1988).

[21] D. Friedan and S. H. Shenker, Nucl. Phys. B281, 509 (1987).

[22] D. Cremades, L. E. Ibanez, and F. Marchesano, JHEP 05, 079 (2004), hep-th/0404229.

[23] M. Bianchi, G. Pradisi, and A. Sagnotti, Nucl. Phys. B376, 365 (1992).

[24] A. Giveon, M. Porrati, and E. Rabinovici, Phys. Rept. 244, 77 (1994), hep-th/9401139.

[25] L. Magnea, R. Russo, and S. Sciuto, Int. J. Mod. Phys. A21, 533 (2006), hep-th/0412087.

[26] M. Bertolini, M. Billo, A. Lerda, J. F. Morales, and R. Russo, Nucl. Phys. B743, 1 (2006), hep-th/0512067.

[27] C.-S. Chu and P.-M. Ho, Nucl. Phys. B550, 151 (1999), hep-th/9812219.

[28] C.-S. Chu, R. Russo, and S. Sciuto, Nucl. Phys. B585, 193 (2000), hep-th/0004183.

Y. Kiem, S.-M. Lee, and J. Park, Nucl. Phys. B594, 169 (2001), hep-th/0008002.

Y. Kiem, D. H. Park, and H.-T. Sato, Nucl. Phys. B601, 27 (2001), hep-th/0011019.

[29] I. Pesando, (2005), hep-th/0505052.

P. Di Vecchia, A. Liccardo, R. Marotta, F. Pezzella, and I. Pesando, (2006), hep-th/0601067.

[30] P. Di Vecchia, Personal communication.

[31] R. R. Metsaev and A. A. Tseytlin, Nucl. Phys. B298, 109 (1988).

[32] P. Di Vecchia, L. Magnea, A. Lerda, R. Marotta, and R. Russo, Phys. Lett. B388, 65 (1996), hep-th/9607141.

A. Frizzo, L. Magnea, and R. Russo, Nucl. Phys. B579, 379 (2000), hep-th/9912183. 